\title{
Linkage of the Actin Cytoskeleton to the Postsynaptic Density via Direct Interactions of Abp1 with the ProSAP/ Shank Family
}

\author{
Britta Qualmann, ${ }^{1}$ Tobias M. Boeckers, ${ }^{2}$ Monika Jeromin, ${ }^{1}$ Eckart D. Gundelfinger, ${ }^{1}$ and Michael M. Kessels ${ }^{1}$ \\ ${ }^{1}$ Department of Neurochemistry and Molecular Biology, Leibniz Institute for Neurobiology, D-39118 Magdeburg, Germany, and ${ }^{2}$ Department of Anatomy \\ and Cell Biology, University of Ulm, D-89081 Ulm, Germany
}

\begin{abstract}
Synaptic contacts contain elaborate cytomatrices on both sides of the synaptic cleft, which are believed to organize and link the different synaptic functions in time and space and can respond to different inner and outer cues with massive structural reorganizations. At the PSD (postsynaptic density), activity-dependent reorganizations of the cortical actin cytoskeleton are hypothesized to play a role in synaptic plasticity. Here, we report on interactions of the F-actin binding protein Abp1 with members of the ProSAP/Shank family: multidomain scaffolding PSD proteins interconnecting glutamate receptors with other synaptic components. Affinity-purification experiments demonstrate that the interactions are mediated by the Abp1 (actin-binding protein 1) SH3 (Src homology 3) domain, which associates with a proline-rich motif that is conserved within the C-terminal parts of ProSAP1(proline-rich synapse-associated protein 1)/Shank2 and ProSAP2/Shank3. The distribution of Abp1, ProSAP1, and ProSAP2 overlaps within the brain, and all three proteins are part of the PSD and are particularly enriched in cortex and hippocampus. Coimmunoprecipitation of endogenous Abp1 and ProSAP2 and colocalization studies of Abp1 and ProSAPs in hippocampal neurons indicate the in vivo relevance of the interactions. Intriguingly, in vivo recruitment assays demonstrate that Abp1 can bind to dynamic F-actin structures and ProSAPs simultaneously, suggesting that Abp1 might link different organizing elements in the PSD. Importantly, different paradigms of neuronal stimulation induce a redistribution of Abp1 to ProSAP-containing synapses. Our data suggest that ProSAPs may serve to localize Abp1 to dendritic spines, thus serving as attachment points for the dynamic postsynaptic cortical actin cytoskeleton. This creates a functional connection between synaptic stimulation and cytoskeletal rearrangements.
\end{abstract}

Key words: Abp1; synaptic scaffold; actin cytoskeleton; ProSAPs/Shanks; postsynaptic density; dynamin; endocytosis; organizing elements

\section{Introduction}

Synapses are highly organized sites of neuronal cell-cell contact and signal transduction that are characterized by intense membrane trafficking. Elements structurally organizing synaptic functions in time and space may include lipid domains, specialized scaffolding proteins, and the cytoskeleton (Gundelfinger et al., 2003). The interplay of cytomatrix components with membrane trafficking processes appears of particular importance for neurogenesis, synaptic transmission, and synaptic plasticity.

The postsynaptic side is characterized by the PSD (postsynaptic density), an electron-dense accumulation of cytoskeletal com-

\footnotetext{
Received Aug. 19, 2003; revised Jan. 14, 2004; accepted Jan. 16, 2004.

This work was supported by Deutsche Forschungsgemeinschaft Grants Qu 116/2-1 and Qu116/2-3 (B.Q.), Bo 1718/2-1 and Bo 1718/2-2 (T.M.B.), and Ke 685/2-1 (M.M.K.), as well as by Kultusministerium Land Sachsen-Anhalt Grant 3451A/0502M. E.D.G. was supported by grants from the Fonds der Chemischen Industrie and European Commission Grant 3451A/0502M. We thank Kathrin Hartung and Gisela Gaede for their technical assistance and Christina Spilker, Thomas Dresbach, Akvile Inciute, and Martin Kreutz for their help with the preparation of primary hippocampal cell cultures.

Correspondence should be addressed to Michael M. Kessels, Department of Neurochemistry and Molecular Biology, Leibniz Institute for Neurobiology, Brenneckestrasse 6, D-39118 Magdeburg, Germany. E-mail: kessels@ifn-magdeburg.de.

DOI:10.1523/JNEUROSCI.5479-03.2004

Copyright $\odot 2004$ Society for Neuroscience $\quad$ 0270-6474/04/242481-15\$15.00/0
}

ponents, cell adhesion and signal transduction molecules, neurotransmitter receptors, and ion channels (Sheng and Sala, 2001). The complex postsynaptic network is able to respond to different stimuli with massive reorganizations of intracellular and of membrane-integrated components. Whereas the surface availability of AMPA receptors, i.e., the size of the activatable pool, is controlled by clathrin-mediated endocytosis (Man et al., 2000), morphology of spines and their alterations depend on the postsynaptic actin cytoskeleton. Together, these synaptic plasticity processes are thought to provide the basis for learning and memory (Matus, 2000; Hering and Sheng, 2002). For both internalization of neurotransmitter receptors and changes of cytoskeletal organization, interplay with PSD scaffolds appears indispensable.

Despite the growing interest, our knowledge about proteins that can functionally interconnect organizing elements with synaptic processes is very limited (Gundelfinger et al., 2003). With syndapins, HIP1R (Huntingtin-interacting protein 1), and Abp1 (actin-binding protein 1), we identified several proteins that can link membrane trafficking processes to the actin cytoskeleton (Qualmann et al., 2000; Qualmann and Kessels, 2002). Mammalian Abp1 (SH3P7, Sparks et al., 1996; HIP55, Ensenat et al., 
1999) interacts with actin filaments via two N-terminal domains, as shown in in vitro reconstitutions (Kessels et al., 2000). In vivo, Abp1 is specifically recruited to newly forming F-actin structures, such as the leading edge of moving and spreading cells, during signaling leading to Racl activation (Kessels et al., 2000).

The C-terminal SH3 (Src homology 3) domain of Abp1 binds to dynamin, the GTPase controlling receptor-mediated endocytosis (Kessels et al., 2001). Consistently, an excess of the SH3 domain of Abp1 blocked endocytosis. This phenotype was rescued by cooverexpression of dynamin or by readdition of the actin-binding modules of Abp1 to the dominant-negatively acting $\mathrm{SH} 3$ domain. The association of Abp1 with endocytic sites appeared to be signal responsive. Abp1 may therefore play a role in the organization of the endocytic machinery and/or coordinate endocytic and cytoskeletal functions (Kessels et al., 2001).

In neurons, Abp1 is much more abundant postsynaptically than presynaptically (Kessels et al., 2001; Yamazaki et al., 2001). However, besides F-actin, no postsynaptic Abpl binding partners are known. Here, we report on the identification of ProSAPs/ Shanks, multidomain PSD-scaffold proteins that directly and indirectly bind to different types of postsynaptic receptors (Sheng and Kim, 2000; Boeckers et al., 2002), as interaction partners for Abp1. We find a direct association with ProSAP/Shank proteins via the $\mathrm{SH} 3$ domain of $\mathrm{Abp} 1$ and confirm this interaction in vivo. Interestingly, Abp1 can associate with dynamic cortical F-actin and ProSAPs/Shanks simultaneously and is incorporated into ProSAP/Shank-positive synapses during synaptic stimulation, suggesting that Abp1-ProSAP/Shank complexes might serve to connect postsynaptic activity to structural plasticity via rearrangements of the postsynaptic actin cytoskeleton.

\section{Materials and Methods}

DNA constructs and recombinant proteins. Constructs encoding glutathione $S$-transferase (GST)-Abp1 and GST-endophilin SH3 domains were described previously (Kessels et al., 2000, 2001). For ProSAP1/Shank2 GST- and green fluorescent protein (GFP) fusion proteins (i.e., GSTProSAP1C and GFP-ProSAP1C), a cDNA fragment encoding for the amino acids 827-1259 was cloned into the appropriate GST- (pGEX4T; Invitrogen, Carlsbad, CA) or GFP- (BD Bioscience, Cambridge, UK) vectors. GST fusion proteins were expressed in Escherichia coli BL21 cells and purified as described previously (Qualmann et al., 1999; Kessels et al., 2000).

Constructs encoding N-terminally myc-tagged Abp $1 \mathrm{SH} 3$ domain and full-length Abp1 have been described previously (Kessels et al., 2001).

Antibodies. Anti-ProSAP1/Shank2 and anti-ProSAP2/Shank3 antibodies, as well as polyclonal rabbit anti-Piccolo antibodies, used in this study have been described previously (Boeckers et al., 1999a; Bockmann et al., 2002; Fenster et al., 2003).

Affinity-purified guinea pig anti-mAbp1 antibodies and rabbit antiGST antibodies were also described previously (Qualmann et al., 1999; Kessels et al., 2000). Monoclonal anti-actin antibody (C4) was purchased from ICN Biochemicals (Costa Mesa, CA), and anti-clathrin heavy-chain antibodies were from Transduction Laboratories (Lexington, KY). Monoclonal anti-synaptophysin antibodies and monoclonal anti-MAP2 (microtubule-associated protein 2) antibodies (clone HM-2) were from Sigma (St. Louis, MO), and monoclonal anti-GFP (B34) and anti-myc (9E10) antibodies were from Babco (Richmond, CA).

Secondary antibodies used in this study include goat anti-mouse peroxidase (Dianova, Hamburg, Germany), goat anti-rabbit peroxidase (Dianova), goat anti-guinea pig peroxidase (ICN Biochemicals), FITC goat anti-guinea pig (ICN Biochemicals), Alexa Fluor 568 goat antimouse (Molecular Probes, Eugene, OR), and Alexa Fluor 568 goat antirabbit (Molecular Probes).

Preparation of cell and tissue extracts. Complete rat brains were prepared from 8-week-old male rats. The brains were frozen immediately in liquid nitrogen. Dissected rat brains were homogenized 1:3 (w/v) in 10
mM HEPES, 1 mM EGTA, and $0.1 \mathrm{~mm} \mathrm{MgCl}_{2}$, $\mathrm{pH}$ 7.4, supplemented with protease inhibitors (complete protease inhibitor tablet, EDTA free; Roche, Mannheim, Germany) containing $150 \mathrm{~mm} \mathrm{NaCl}$ (for immunoprecipitation (IP) and coprecipitation assays with immobilized GSTSH3 domains) or $10 \mathrm{~mm} \mathrm{NaCl}$ (for coprecipitation assays with immobilized GST-ProSAP1C) with an ultra turrax at 20,000 rpm for $10 \mathrm{sec}$ and subsequently supplemented with Triton X-100 (1\% final). The homogenate was centrifuged at $150,000 \times g$ for $45 \mathrm{~min}$, yielding supernatant $\mathrm{S} 3$ and pellet $\mathrm{P} 3$. To obtain rat brain extracts for the coprecipitation assays with immobilized GST-SH3 domains, pellet $\mathrm{P} 3$ was extracted with radioimmunoprecipitation assay buffer $(0.1 \%$ SDS, $0.5 \%$ sodium desoxycholate, $1 \%$ Nonidet P-40, $50 \mathrm{~mm}$ Tris- $\mathrm{HCl}, \mathrm{pH} 8.0$, and $150 \mathrm{~mm} \mathrm{NaCl}$ ) for $30 \mathrm{~min}$ and recentrifuged at $150,000 \times g$ for $45 \mathrm{~min}$. The supernatant obtained was combined with S3. All procedures were performed at $4^{\circ} \mathrm{C}$.

COS-7 cells were transfected with a construct encoding GFP-tagged ProSAP1C using the LipofectAMINE PLUS transfection reagent method according to the instructions of the manufacturer (Invitrogen). Cell lysates were prepared $48 \mathrm{hr}$ after transfection. Cells were harvested and resuspended in $30 \mu \mathrm{l}$ of lysis buffer [1\% Triton X-100 in PBS supplemented with protease inhibitors (complete protease inhibitor tablet, EDTA free; Roche)] and incubated for $20 \mathrm{~min}$ on ice. The samples were then spun for $20 \mathrm{~min}$ at $14,000 \times g$ at $4^{\circ} \mathrm{C}$.

Tissue fractionation. Tissue fractionation was performed essentially as described by Wyneken et al. (2001) with slight modifications. Cortices and hippocampi of four rat brains were pooled and immediately homogenized in $320 \mathrm{~mm}$ sucrose and $5 \mathrm{~mm}$ HEPES, pH 7.4. Cell debris and nuclei were removed by $1000 \times g$ centrifugation, yielding a low-speed supernatant $(\mathrm{S} 1)$, which was centrifuged at $12,000 \times g$ for $15 \mathrm{~min}$. The resulting supernatant S2 was used for generating a microsomal pellet $(\mathrm{PM})$ and a ultra-high-speed supernatant $(\mathrm{SM})$ by centrifugation at $100,000 \times g$ for $1 \mathrm{hr}$, whereas the resulting pellet P2 (crude membrane fraction) was used for isolation of synaptosomes by a $0.85 / 1.0 / 1.2 \mathrm{M}$ sucrose step gradient. Myelin, light membranes, and synaptosomes were isolated at the different sucrose interfaces and mitochondria as a pellet. Synaptosomes were subjected to osmotic shock in $1 \mathrm{~mm}$ Tris/HCl, $\mathrm{pH}$ 8.1 , for $30 \mathrm{~min}$ and then fractionated on a second sucrose step gradient, from which the synaptic junctions were isolated at the 1.0/1.2 $\mathrm{M}$ sucrose interface. Synaptic junctions were subsequently extracted twice with Triton X-100 for $15 \mathrm{~min}$, and the final PSD fraction was collected by centrifugation at 33,000 $\times g$ for $30 \mathrm{~min}$ according to Wyneken et al. (2001). All experimental steps were performed at $0-1^{\circ} \mathrm{C}$. Protein $(10 \mu \mathrm{g})$ of each fraction was loaded on 5-20\% polyacrylamide gels, and the subcellular protein distribution was analyzed by immunoblotting.

Blot overlay analysis and coprecipitation assays. Blot overlay experiments were performed with recombinant GST-Abp1 SH3 domain on rat brain fractions, as well as COS-7 cell extracts transfected with GFPProSAP1C according to the procedure described previously (Kessels and Qualmann, 2002).

Coprecipitations of rat brain proteins interacting with GSTProSAP1C or GST alone were performed with $0.25 \mathrm{mg}$ of rat brain extract containing 10 or $150 \mathrm{~mm} \mathrm{NaCl}$ final according to Qualmann and Kelly (2000). Bound proteins were separated on 9.5\% SDS-PAGE, blotted to nitrocellulose, and probed for with affinity-purified guinea pig antibodies against Abp1 (GP1).

Immobilized GST, GST-Abp1 SH3 domain, and GST-endophilin SH3 domain, respectively, were incubated with aliquots of the combined rat brain extracts overnight at $4^{\circ} \mathrm{C}$. After several washes with IP buffer $(10$ mM HEPES, pH 7.4, 1 m EGTA, $0.1 \mathrm{~mm} \mathrm{MgCl}_{2}, 150 \mathrm{~mm} \mathrm{NaCl}$, and $1 \%$ Triton X-100), specifically bound proteins were eluted with a glutathione-containing buffer according to Qualmann et al. (1999). Eluates were separated on 9.5\% SDS-PAGE and analyzed by immunoblotting using antibodies against ProSAP1 and ProSAP2.

Immunoprecipitations. Affinity-purified anti-mAbp1 antibodies (GP5) (Kessels et al., 2000) or unrelated guinea pig IgGs were immobilized on protein $\mathrm{G}$ Sepharose (Amersham Biosciences, Buckinghamshire, UK) in the presence of 5\% BSA. After several washes with IP buffer, the resin was incubated overnight with $0.44 \mathrm{mg}$ of rat brain extract S3 adjusted to a final concentration of $2 \mathrm{mg} / \mathrm{ml}$ protein in IP buffer containing $50 \mathrm{~mm} \mathrm{NaCl}$ final. Beads were washed four times with IP buffer $(50 \mathrm{~mm}$ 
$\mathrm{NaCl}$ ). Bound proteins were eluted with SDS sample buffer. All procedures were performed at $4^{\circ} \mathrm{C}$. Eluates were separated on $8 \%$ SDS-PAGE and analyzed by immunoblotting using rabbit anti-ProSAP2 antibodies.

Immunohistochemistry. Immunocytochemical staining was performed using $7 \mu \mathrm{m}$ microtome sections from rat brains, which were fixed by immersion in Bouin's fluid for $48 \mathrm{hr}$, dehydrated, and embedded in paraplast. Abp1 and ProSAP2 were detected with guinea pig anti-Abp1 and anti-ProSAP2 polyclonal antibodies diluted 1:1000 to 1:3000. After incubation of the sections with the primary antibody overnight, the binding was visualized by incubating the sections with biotin-labeled goat anti-guinea pig IgG (Jackson ImmunoResearch, West Grove, PA). The sections were then incubated for $30 \mathrm{~min}$ with a preformed complex of biotin peroxidase-streptavidin (Jackson ImmunoResearch), and peroxidase activity was revealed by applying the color reagent 3,3-diaminobenzidine $/ \mathrm{H}_{2} \mathrm{O}_{2}$ (Sigma) for $5 \mathrm{~min}$. For control purposes, the primary antibodies were omitted, and the sections were merely incubated with the secondary antibodies and the reagents for the color development.

In situ hybridization. In situ hybridization was performed essentially as described previously (Kreutz et al., 1997). Transcripts encoding Abp1 were detected with $S^{35}$-labeled cDNA antisense oligonucleotides purchased from MWG-Biotech (Ebersberg, Germany).

The Abp1 probe used was as follows: $5^{\prime}$-TGC AGC GCC GGC CCG TTC CGG CTC AGG TTC ACC GCC AT- $3^{\prime}$. Controls were performed as follows: (1) omission of the antisense oligonucleotide, (2) posthybridizational washing steps above the calculated melting point of the hybrid, (3) hybridization with the corresponding sense oligonucleotide, and (4) hybridization in the presence of 100 -fold excess of unlabeled oligonucleotide. In none of these cases was any specific labeling observed.

Cell culture and immunofluorescence microscopy. NIH3T3 cells were maintained in DMEM containing 10\% fetal bovine serum. Primary hippocampal cultures were prepared and grown on poly-D-lysine-coated glass coverslips (Kessels et al., 2001). Transfections with myc-Abp1 were performed as described previously (Fenster et al., 2003).

NIH3T3 cells were fixed in 4\% paraformaldehyde for $15 \mathrm{~min}$ at room temperature. Primary hippocampal neurons were fixed for 10-15 min. Stimulations of primary hippocampal cells kept in culture for $17 \mathrm{~d}$ were performed by adding glutamate directly to the medium (100 $\mu \mathrm{M} ; 10 \mathrm{~min}$ of incubation) before fixation. In some experiments, $\mathrm{KCl}$ stimulations were performed for comparison (in mM: $90 \mathrm{KCl}$ in $20 \mathrm{HEPES}, \mathrm{pH} 7.5$, containing $50 \mathrm{NaCl}, 15 \mathrm{MgCl}_{2}, 250$ glucose, and $1.5 \mathrm{CaCl}_{2} ; 3$ min of incubation). In other experiments, NMDA-, AMPA-, and kainite-type glutamate receptors (Sigma-Aldrich, Steinheim, Germany) were inhibited with $50 \mu \mathrm{M}[5 \mathrm{R}, 10 \mathrm{~S}]-(+)-5$-methyl-10,11-dihydro-5H-dibenzo [a,d] cyclohepten-5,10-imine maleate [(+)-MK-801], $50 \mu \mathrm{M} \mathrm{D}(-)-2-$ amino-5-phosphonopentanoic acid (AP-5), $50 \mu \mathrm{M}$ 1,2,3,4-Tetrahydro6-nitro-2,3,-dioxo-benzo[f] quinoxaline-7-sulfonamide disodium salt (NBQX), and $100 \mu \mathrm{M}$ CNQX for $10 \mathrm{~min}$, and cells were fixed $20 \mathrm{~min}$ thereafter. After an incubation with $25 \mathrm{~mm}$ glycine in PBS for $15 \mathrm{~min}$ (quench of fixative), cells were permeabilized and blocked for $1 \mathrm{hr}$ in $2 \%$ BSA, $10 \%$ horse serum, and $0.02 \%$ saponin in PBS (block solution). Incubations with antibodies were performed according to Kessels et al. (2001). Images were recorded digitally using a Leica (Nussloch, Germany) TCS NT laser confocal microscope with a Leica TCS software package and processed using Adobe Photoshop software (Adobe Systems, San Jose, CA).

The examination of the Abp 1 and ProSAP localization in stimulated, resting and inhibited neuronal cultures is based on randomly recorded confocal images from the respective samples. The quantitative examinations were performed following the principles described by Dick et al. (2001). The images were mixed and encoded by numbers. Each image was then quantitatively analyzed by the following procedure. First, the signal intensities of the nonsynaptic, neuritic Abp1 staining of 20-44 image points were measured, averaged, and subtracted from the Abp1 image data. Only areas without any immunostaining of ProSAP, which is known to be localized postsynaptically, were selected to avoid any overlap with potential synaptic areas. Second, the contrast of the ProSAP1 immunostainings was slightly raised by subtracting the 50 lowest gray values in all images. This value represented unspecific background, as detected in several nonsynaptic areas of the ProSAP1 immunostainings.
Third, the pattern of ProSAP immunostaining was then overlaid with dots of average synaptic size to define synaptic areas. The thus defined synaptic areas were merged with the Abp 1 immunostainings and examined for the presence of Abp1 immunosignals within these areas. The number of Abpl-enriched synaptic areas per total of synaptic areas defined by the ProSAP1 immunolabeling was determined for each image. The images were then decoded, the values of corresponding examinations were averaged, and the total number of synaptic areas examined was counted. SDs represent data from independent images. Statistic analysis was done by applying a two-sided $t$ test, and $p$ value thresholds are according to the $t$ test table $(0.05,0.02,0.01,0.002$, and 0.001$)$.

Recruitment and cell perforation experiments in fibroblasts. Resting NIH3T3 fibroblasts transfected with GFP-ProSAP1C and myc-tagged Abp1 full-length or myc-tagged Abp1 SH3 domain were activated by replating the cells onto fibronectin-coated coverslips and additional stimulation with $300 \mathrm{ng} / \mathrm{ml} \mathrm{PMA} \mathrm{(Sigma)} \mathrm{and} 5 \mathrm{ng} / \mathrm{ml}$ human recombinant PDGF (Sigma), as described previously (Kessels et al., 2000). The cells were then washed with warm PBS and subsequently either briefly perforated with $0.02 \%$ saponin (Sigma) for $5 \mathrm{sec}$ or directly fixed with $4 \%$ paraformaldehyde in PBS, pH 7.4, containing $0.9 \mathrm{~mm} \mathrm{CaCl}_{2}$ and $0.5 \mathrm{~mm}$ $\mathrm{MgCl}_{2}$ for $20 \mathrm{~min}$. The cells were then processed for immunofluorescence microscopy using anti-myc antibody 9E10 and Alexa Fluor 568 goat anti-mouse antibody conjugates. Images were recorded digitally using a Leica TCS NT laser confocal microscope with a Leica TCS software package and processed using Adobe Photoshop software.

For quantitative analyses, the number of GFP-ProSAP1C-expressing cells per coverslip was quantified live $45 \mathrm{~min}$ after replating using an inverted Eclipse TE300 fluorescence microscope (Nikon, Tokyo, Japan). Cells were then perforated, fixed, and processed for fluorescence microscopy. Cells with remaining GFP-ProSAP1C signal were counted again. Several independent experiments were performed, and the extent of GFP-ProSAP1C retention was expressed as percentage of the number of observed GFP-ProSAP1C-positive cells after extraction per total number of GFP-ProSAP1C-expressing cells. SDs were calculated from independent experiments.

\section{Results}

\section{Identification of ProSAPs/Shanks as binding partners of the Abp1 SH3 domain}

Abp1 is a signal-responsive component of the actin cytoskeleton that was shown recently to link cytoskeletal functions to other cellular processes and may therefore be an important organizing element (for review, see Qualmann and Kessels, 2002). Tight coordination of different cellular functions, such as membrane trafficking, signaling pathways, and actin cytoskeletal dynamics, may be of special importance in synaptic structures (for review, see Gundelfinger et al., 2003). To identify synapse-specific proteins that may serve as contact sites for the cytoskeletal functions of Abp1, we performed cell fractionation studies and synaptic junction preparations and screened for Abp1-binding proteins by using the blot overlay technique (Fig. 1a).

The intense double band at $\sim 78 \mathrm{kDa}$ was identified as synapsins and that of $100 \mathrm{kDa}$ as dynamin 1 (Fig. $1 a$, asterisks indicate previously identified Abp1-binding partners) (analysis not shown) (cf. Kessels et al., 2001). Dynamin 1 is readily detected in the different supernatants and in the microsomal, crude, and light membrane fractions, as well as in the synaptosomal fraction. The amount of dynamin 1 is, however, reduced in the course of the preparation of PSDs. The distribution of synapsins is similar, except that they are still present in synaptic junctions and in the PSD fraction. The slight increase of synapsins in the PSD fraction probably reflects the cytoskeletal association of these synaptic vesicle proteins. One protein band detected at very high molecular weight ( $\sim 500 \mathrm{kDa})$ (Fig. 1a, asterisk) and strongly enriched in the PSD fraction was identified as the cytomatrix protein Piccolo (Fenster et al., 2003). 


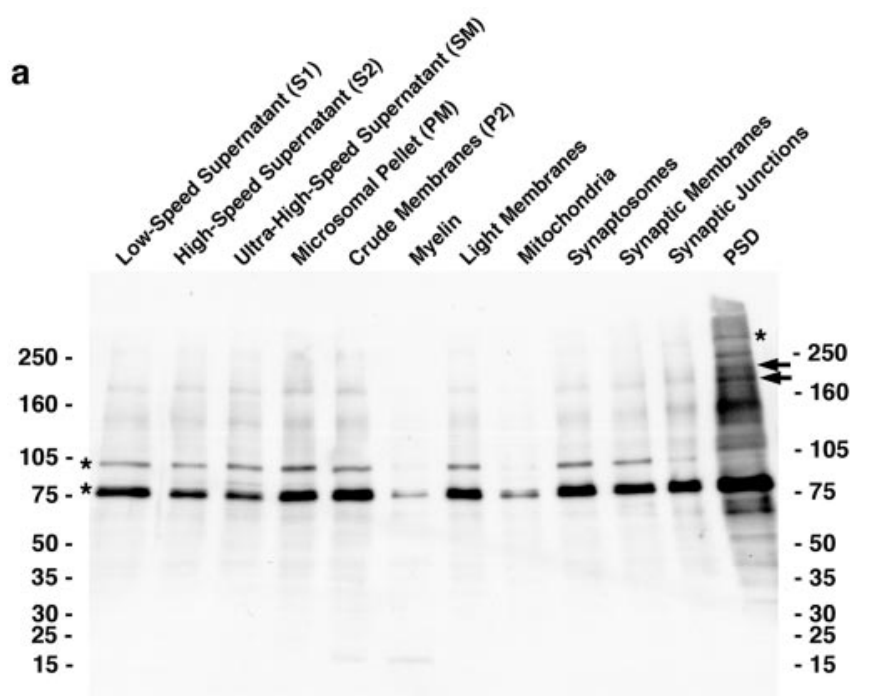

b

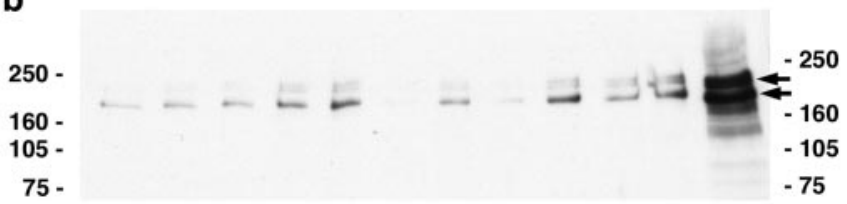

Figure 1. Detection of a PSD-enriched Abp1 interaction partner, which comigrates with ProSAP1, in blot overlays. $a$, Rat brain cell fractionation samples (2.5 $\mu \mathrm{g}$ protein each) were blotted to nitrocellulose and overlaid with a GST-SH3 domain fusion protein of Abp1 as a probe. Bands recognized by the probe were visualized by anti-GST antibodies. The asterisks mark previously identified Abp1-binding proteins (synapsins at $78 \mathrm{kDa}$, dynamin at $100 \mathrm{kDa}$, and Piccolo far above $250 \mathrm{kDa}$ ), and the arrows mark examples of thus far unknown Abp1-binding proteins enriched in the PSD preparation. $b$, ProSAP1 immunoreactivity migrates at positions identical with those highlighted by the Abp1 probe in blot overlays (arrows).

Additionally, the Abp1 SH3 domain probe detected multiple bands at $\sim 180 \mathrm{kDa}$ that were readily detectable in the synaptic junction preparation and strongly enriched in the PSD preparation (Fig. 1a, arrows). We thus reasoned that these bands must correspond to PSD-specific proteins. Immunoblotting analyses with anti-ProSAP antibodies revealed that distribution and band patterns of ProSAP/Shank proteins are similar to the bands detected by the Abp1 SH3 domain (Fig. 1b).

To test whether the observed bands may indeed correspond to proteins of the ProSAP family, we performed affinitypurification experiments with immobilized Abp1 SH3 domain and immunoblotted the eluates. ProSAP/Shank proteins represent a protein family currently comprising three isoforms encoded by different genes and multiple splice variants of these isoforms (for review, see Boeckers et al., 2002). ProSAP1 (Boeckers et al., 1999a) corresponds to CortBP1 (cortactin-binding protein 1) (Du et al., 1998), Shank2 (Naisbitt et al., 1999; Tu et al., 1999), and Spank3 (Tobaben et al., 2000). The ProSAP2 isoform (Boeckers et al., 1999b) corresponds to Shank3 (Naisbitt et al., 1999; Tu et al., 1999) and Spank1 (Tobaben et al., 2000). The third isoform is referred to as Shank1 (Naisbitt et al., 1999; Tu et al., 1999), Synamon (Yao et al., 1999), SSTRIP (somatostatin receptor interactive protein) (Zitzer et al., 1999), and Spank2 (Tobaben et al., 2000). We tested Abp1 binding with both antiProSAP1 (Fig. 2a) and anti-ProSAP2 (Fig. $2 b$ ) antibodies. Both proteins were effectively bound by the Abp $1 \mathrm{SH} 3$ domain but not by GST alone, which was used as a control.

Highly related SH3 domains from different proteins often

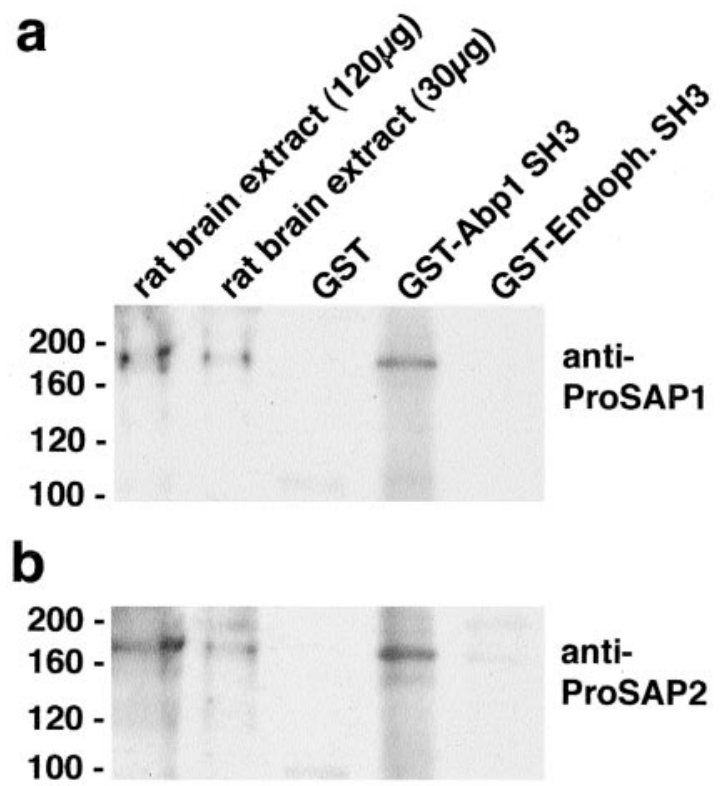

Figure 2. Identification of ProSAP1 and ProSAP2 as binding partners for the Abp1 SH3 domain in rat brain. Affinity purifications of proteins interacting with the immobilized $\mathrm{SH} 3$ domains of Abp1, of endophilin I, and with GST alone in rat brain extracts. Immunoblot analyses of the precipitated material with antibodies against ProSAP1 and ProSAP2 demonstrate that a GST fusion protein of the Abp $1 \mathrm{SH} 3$ domain, but not the related endophilin SH3 domain or GST alone, binds to both ProSAP1 $(a)$ and ProSAP2 $(b)$ in rat brain extracts.

show similar binding specificity. For example, the sets of synaptic binding partners for endophilin and Abp 1 seem to be very similar in vitro (Kessels et al., 2001). The SH3 domains of both proteins specifically bind to the GTPase dynamin, to synaptojanin, and to synapsins and differ merely in the binding affinities to these proteins. Interestingly, although the endophilin $\mathrm{SH} 3$ domain shows considerable homology to the Abp1 SH3 domain, it almost did not recognize ProSAP1 (Fig. $2 a$ ) or ProSAP2 (Fig. $2 b$ ). Thus, the capability to associate with ProSAPs rather appears to be a specialty of Abp1.

The Abp1 SH3 domain binds to the C-terminal part of ProSAP1, which contains an SH3 domain-binding motif conserved between ProSAP1 and ProSAP2

The sequence homology between ProSAP1 and ProSAP2 is limited. A comparative sequence analysis of ProSAP1 and ProSAP2 revealed a highly conserved motif within the otherwise not well conserved C-terminal halves of the proteins. This motif may serve as binding site for the Abp1 SH3 domain (Fig. 3a). To test this hypothesis experimentally, we produced a GST fusion protein encompassing the ProSAP1 C-terminal region (ProSAP1C) and immobilized it on beads. This fusion protein specifically affinity-purified Abp1 from rat brain extracts under different salt conditions, as detected by immunoblotting analyses with anti-Abp1 antibodies (Fig. $3 b$ ). This experiment shows that the C-terminal part of ProSAP1 indeed contains an Abp1 binding site.

To confirm that our results represent a direct association of Abp1 with the C-terminal region of ProSAP1, we generated a GFP fusion protein of this part of the ProSAP molecule, overexpressed it in COS-7 cells, and subjected the homogenates of these cells to blot overlay experiments using the Abp1 SH3 domain as a probe. The Abp1 SH3 domain directly and specifically bound to GFP-ProSAP1C (Fig. 3c). 

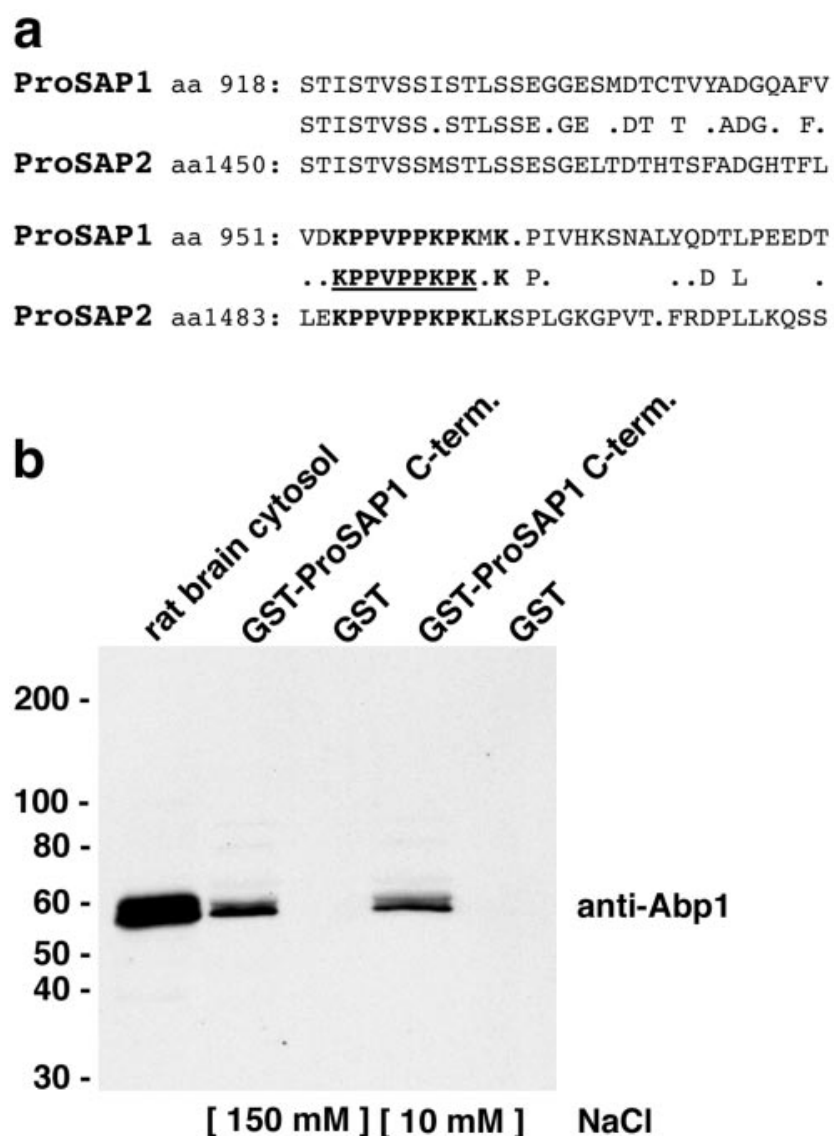

C

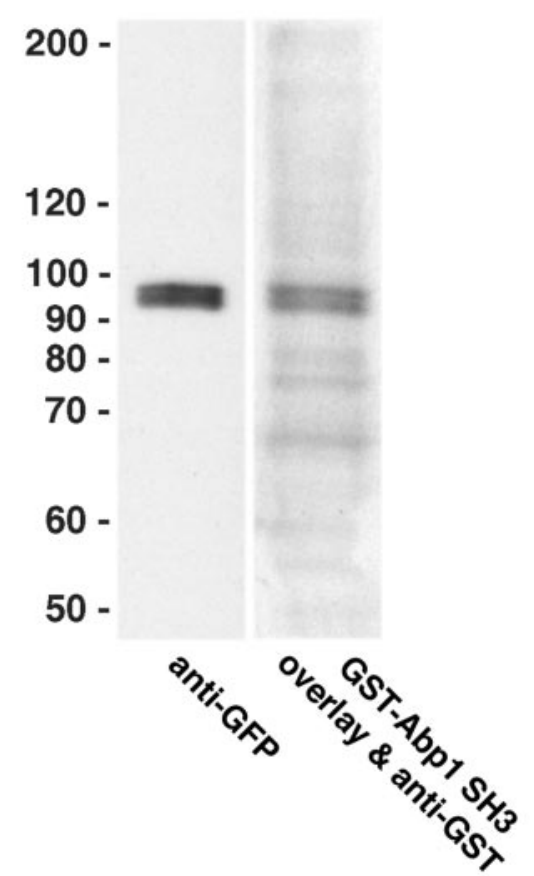

Figure 3. Abp1 interacts directly with the C terminus of ProSAP. a, Alignment of ProSAP isoforms reveals an $\mathrm{SH} 3$ domain recognition interface (underlined), which is strongly conserved among ProSAP1 and ProSAP2 and is located in the C-terminal halves of the proteins. b, Affinity purifications of rat brain proteins interacting with a GST fusion protein of the $C$ terminus of ProSAP1C. GST-ProSAP1C, but not GST alone used as a control, coprecipitated Abp1 from rat brain extracts at conditions of different ionic strength. Equal amounts of fusion proteins and brain extracts $(0.25 \mathrm{mg}$ of brain protein per pull down) were used. Starting material $(30 \mu \mathrm{g})$ was loaded for comparison. Lanes 2 and 3, IP buffer with $150 \mathrm{~mm}$ salt; lanes 4 and 5, IP buffer with $10 \mathrm{~mm}$ salt. c, Blot overlay analysis of extracts from GFP-ProSAP1C-transfected COS-7 cells

\section{Abp1 and ProSAPs/Shanks show overlapping expression} patterns in rat brain sections

To examine whether Abp1 and ProSAPs show overlapping expression patterns in the brain, we next compared the distribution of ProSAP1, ProSAP2, and Abp1 by comparative in situ hybridizations of rat brains at different developmental stages and by immunohistochemical analyses. Because the distribution of ProSAP1 is already well documented (Boeckers et al., 1999a), only Abp1 and ProSAP2 expression is displayed in Fig. 4. In situ hybridization experiments with Abpl-specific oligonucleotide probes revealed a strong mRNA expression in all brain areas investigated. During development, especially cortex, hippocampus, and cerebellum show marked Abpl mRNA levels (Fig. 4a). The expression pattern is in good agreement with that of ProSAP1 and/or ProSAP2 mRNA (Boeckers et al., 1999a,b; Böckers et al., 2001). The early appearance of high Abp1 mRNA levels suggests a role of Abp1 in early brain development and/or synaptogenesis.

Immunohistochemical staining of frontal sections of the brain (Fig. $4 b$, left side) revealed a strong expression of Abpl as well as of ProSAP2 in the cerebral cortex. Virtually all cortical layers are stained. Also, ProSAP1 is highly expressed throughout the cerebral cortex (Boeckers et al., 1999a). Despite the fact that Abp1 and ProSAP1 and ProSAP2 are coexpressed in the cortex, slight differences in the distribution of the highest expression levels within the cortex may exist. Both proteins show a very high expression in layer 1 of the cortex, but whereas ProSAP2 staining is also quite intense in cortical layers 5 and 6, Abp 1 is more evenly distributed in the deeper layers of the cortex.

In the hippocampus, Abp 1 is readily detected in the CA1-CA4 regions, and a particularly strong expression can be observed in the dentate gyrus. Figure $4 e$ shows the Abp 1 distribution in the CA3 region of the hippocampus in higher magnification. ProSAP2 (as ProSAP1) is also expressed in the hippocampus. It is relatively uniformly expressed in neurons of the hippocampus. It lacks the strong accumulation within the dentate gyrus seen for Abp1 (Fig. 4, b, right side, d) (for ProSAP1 distribution, see Boeckers et al. 1999a).

Thalamic and hypothalamic nuclei are moderately stained for Abp1 (Fig. 4, b, left side, $c$ ). Although they show a more intense ProSAP2 expression (Fig. $4, b$, right side, $d$ ), the staining pattern of ProSAP1 in thalamus and hypothalamus appears to be quite similar to that of Abp1 (for ProSAP1 distribution, see Boeckers et al., 1999a).

Sagittal sections of rat brains (Fig. $4 c, d$ ) additionally showed that the molecular and the granular cell layer of the cerebellum are intensely labeled for both Abp1 (Fig. 4c) and ProSAP2 (Fig. $4 d$ ). The sagittal section immunostained for Abp1 also displayed a marked expression of Abp1 in the olfactory bulb (Fig. 4c). Furthermore, the caudate putamen shows a strong expression of both proteins (Fig. $4 c, d$ ). In contrast, the brainstem shows relatively little staining for both proteins. The specificity of the immunostainings was verified by controls, in which the primary antibody was omitted. Figure $4 f$ shows the control accompanying the anti-Abp1 immunostaining in the $\mathrm{CA} 3$ region (Fig. 4e). Furthermore, specificity of the anti-Abp1 immunolabeling is strongly supported by the fact that the detected labeling patterns were consistently observed with two different anti-Abp1 antibodies (data not shown).

with a GST fusion protein of the SH3 domain of Abp 1 demonstrate that the interaction between the two proteins is direct. The Abp1 probe (right lane) readily detected the recombinant ProSAP1C, visualized in the left lane by an immunoblotting with anti-GFP antibodies. 


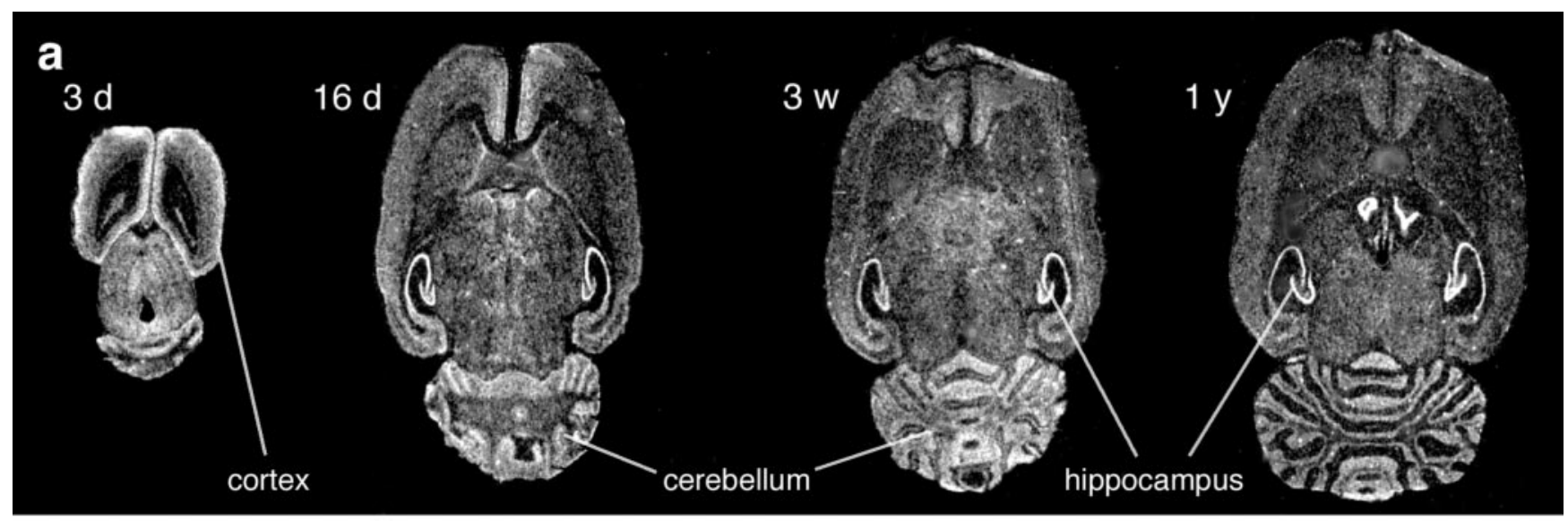

b
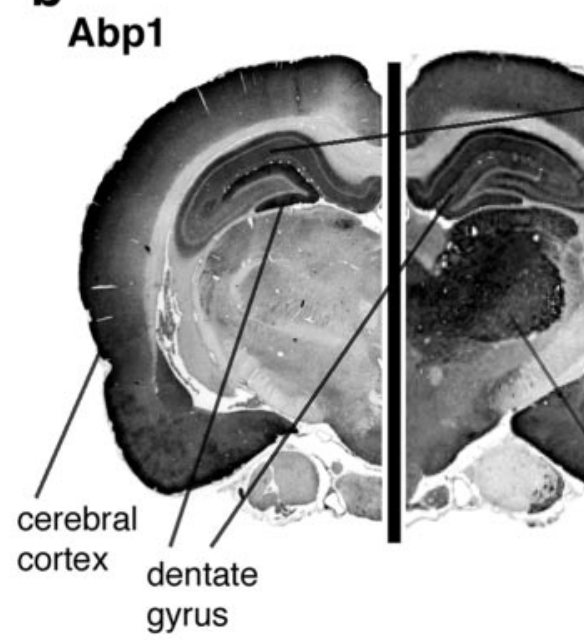

2 gyrus
ProSAP2 hippocampus cerebral cortex

thalamic \& hypothalamic nuclei
C

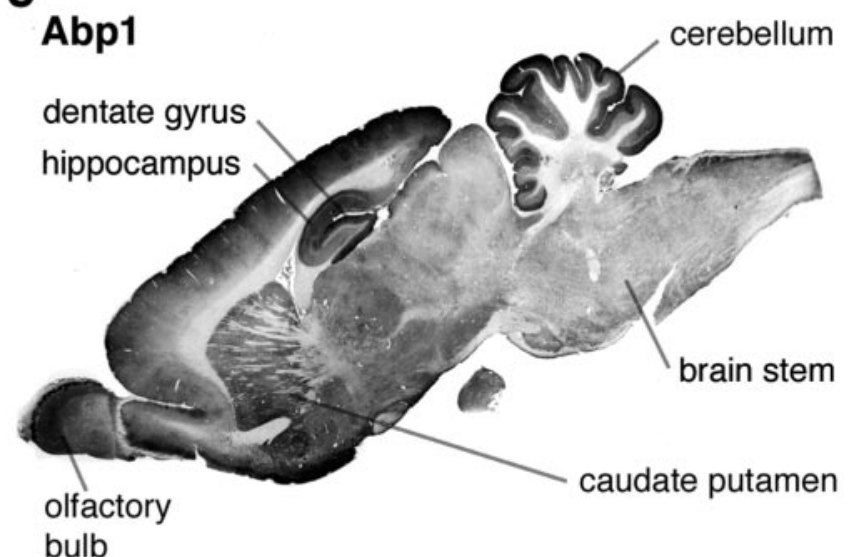

d

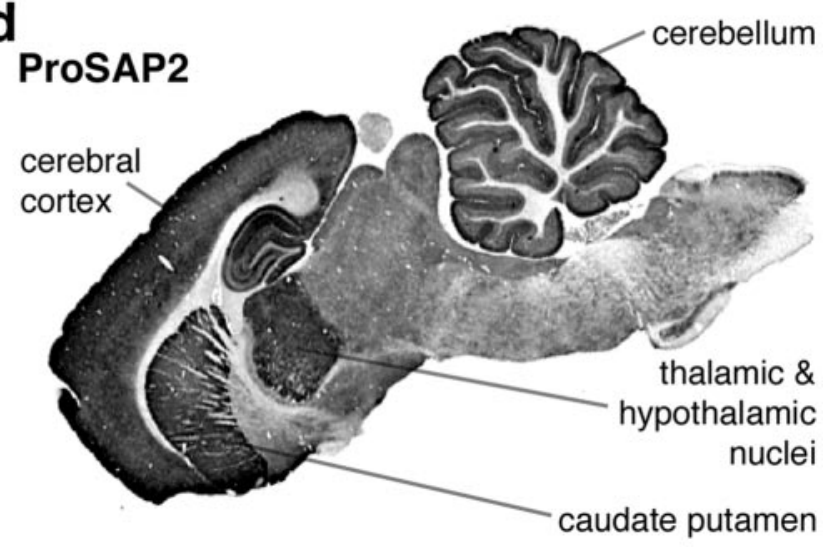

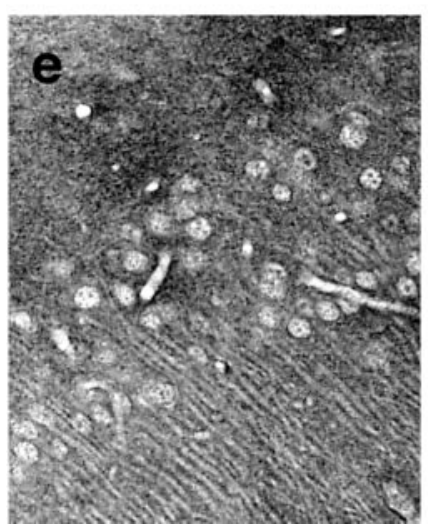

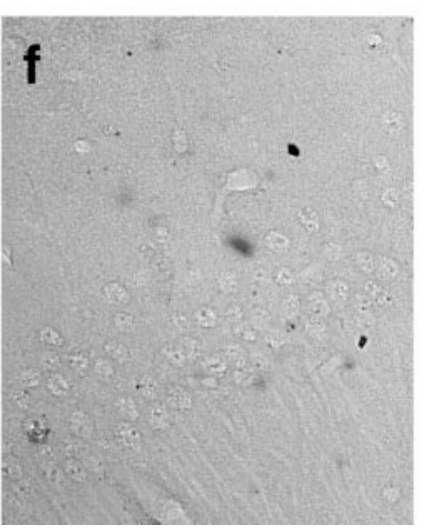

Figure 4. Expression of Abp1 and ProSAP2 in rat brain. $a$, In situ hybridization with an Abp1-specific oligonucleotide at different developmental stages shows the early expression of the transcript in all brain areas depicted. High expression levels are especially seen in the cortex, hippocampus, and cerebellum. $b-d$, Immunohistochemical stainings of rat frontal ( $b$ ) and sagittal $(c, d)$ brain sections with ProSAP2- and Abp1-specific antibodies also show high protein levels of Abp1 in cortex, hippocampus, cerebellum, and caudate putamen, with a remarkable high expression in neurons of the dentate gyrus. ProSAP2 is coexpressed with Abp1 in many areas of the brain. $e$, Anti-Abp1 staining within the CA3 region of the hippocampus at $40 \times$ magnification. $f$, Control section parallel to that shown in $e$ with the primary antibody omitted.

Our data show that Abp1 expression on the one hand and ProSAP1 and/or ProSAP2 expression on the other hand overlap in wide areas of the brain. The observation that expression levels of Abp1 relative to ProSAP2 may vary to some extent may not be surprising. This might be explained by Abp1 binding to several members of the ProSAP/Shank family, as well as by the existence of other ProSAP-interacting cytoskeletal adaptor proteins with Abp1-related functions but different distributions.
In mature neurons, $\mathrm{Abp} 1$ and ProSAPs/Shanks partially colocalize at the subcellular level

Because Abpl and ProSAPs are coexpressed in many areas of the brain, we next asked whether Abp1 and ProSAPs would also colocalize at the subcellular level and performed double-labeling experiments in primary hippocampal cultures (Fig. 5). Abp1 immunostaining was detected in neurites, but some staining was also found in the cell bodies of mature neurons (Fig. $5 a, d$ ). The 

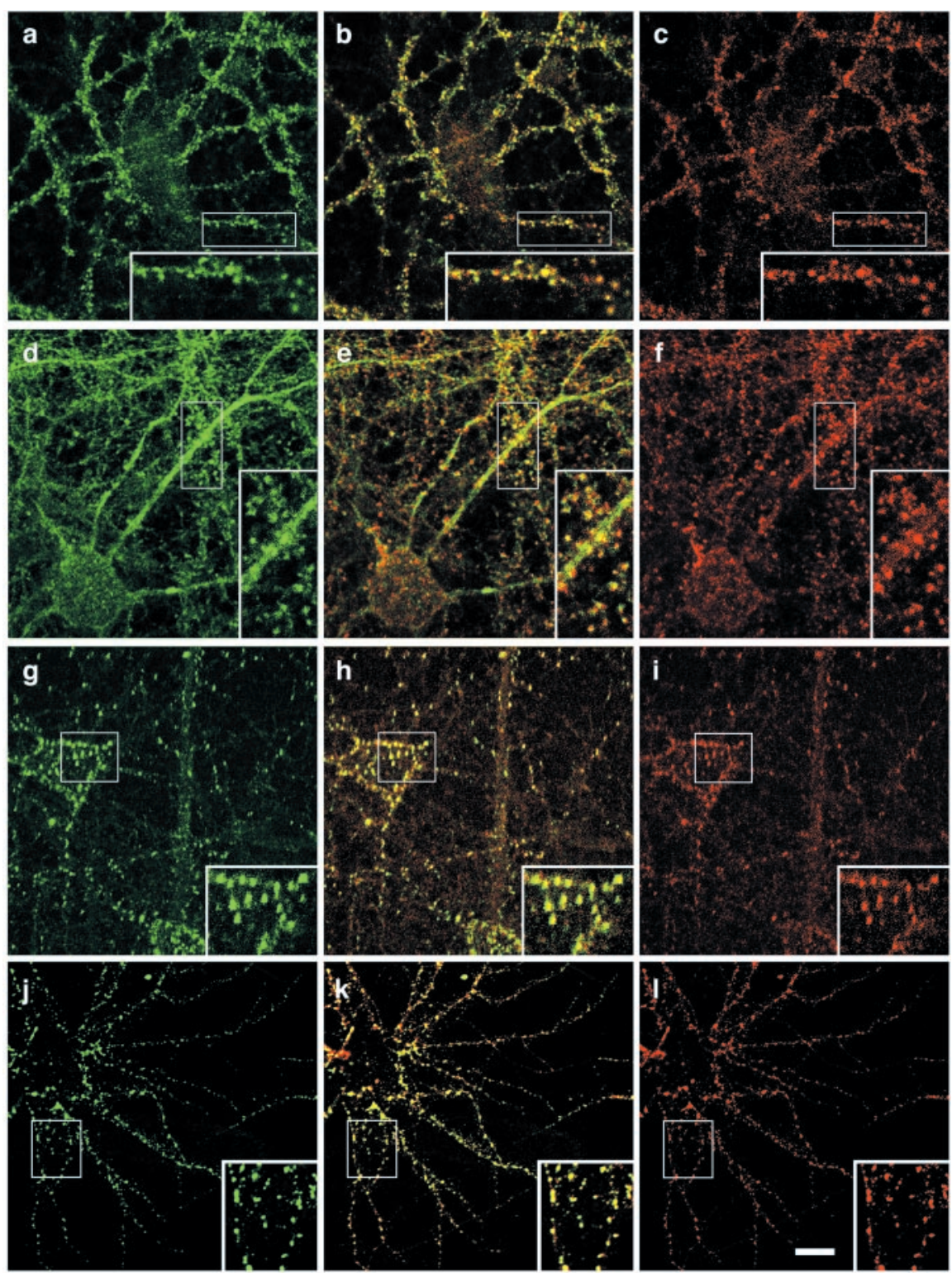

Figure 5. Colocalization of Abp1 with ProSAP1 and ProSAP2 in hippocampal neurons by confocal immunofluorescence microscopy. a, d, Anti-Abp1 staining outlines neurites and displays punctate accumulations adjacent to neurites in primary hippocampa neurons kept in culture for $26 \mathrm{~d}(a-c)$ and $17 \mathrm{~d}(d-f)$, respectively. $a-f$, The immunostaining for Abp1 $(a, d)$ and ProSAP2 (c), as well as ProSAP1 $(f)$ overlaps at synapses, as seen in the merged images $(b, e) . g-i$, The spatial distribution for ProSAP1 $(g, j)$ and ProSAP2 $(i, l)$ exhibits a complete overlap $(h, k)$ in hippocampal neurons throughout neuronal development in culture ( $g$-i, cells kept in culture for $9 \mathrm{~d} ; j-l$, cells kept in culture for $17 \mathrm{~d}$ ). Insets show a clear overlap of the synaptic staining of Abp 1 and ProSAPs, as well as of ProSAP1 and ProSAP2 at higher resolution (twofold enlargements of boxed areas in $a-l$ ). Scale bar, $10 \mu \mathrm{m}$.

\section{Abp1 is localized to the}

dendritic/postsynaptic compartment of neurons and is part of the PSD

We showed previously that Abp1 can interact with proteins that are mainly presynaptically localized and involved in presynaptic membrane trafficking processes, such as dynamin, synapsins, and Piccolo (Kessels et al., 2001; Fenster et al., 2003). We here wanted to formally prove that Abp1 is also localized to the dendritic compartment and that the observed ProSAP colocalization (Fig. 5) indeed represents a postsynaptic localization of Abp1. Therefore, primary hippocampal cultures were costained with anti-Abp1 and anti-MAP2 antibodies (Fig. 6). MAP2 is specifically localized to microtubules of dendrites and is absent from axons. Figure 6 demonstrates that MAP2-positive dendrites $(a)$ are also positive for Abp1 $(b, c)$.

Transfection of primary hippocampal neurons with myc-tagged Abp 1 full-length constructs (Fig. $6 f$ ) allows to exclude the theoretical possibility that the observed Abp1 signal colocalizing with the MAP2 immunosignal represents Abp1-positive axons running along and contacting the MAP2-positive dendrites. Myc-Abp1 (Fig. $6 f$ ) is present in the numerous dendrites extending from the cell body. The Abp1 immunoreactivity was observed throughout the dendrites and displayed a good overlap with the MAP2 signal (Fig. $6 d, e$ ). Interestingly, it was also prominent in small structures protruding from the MAP2-positive dendrites (arrows). These structures are likely to represent postsynaptic spines, which seem to contain high amounts of Abp1 in their heads (Fig. 6d-f, high-magnification insets).

This is in line with the results of the ProSAP colocalization studies (Fig. 5), which show that virtually all neurites protruding from neuronal cells bodies were Abp1 positive. Therefore, Abpl is not restricted to the axonal and presynaptic compartment but, like F-actin, is also highly

immunostaining was not restricted to dendrites but was also present in axons (Fenster et al., 2003). Additionally, strong Abp1 accumulations in dot-like structures protruding from neurites were detected (Fig. 5a,d). Because these structures were also positive for ProSAP1 (Fig. 5c) and ProSAP2 (Fig. 5f), respectively, as seen well in the merged images (Fig. $5 b, e$ ) and the twofold enlargements shown in each panel (Fig. $5 a-f$ ), we concluded that these sites of Abp1 accumulation represent ProSAP1- and ProSAP2-positive synapses.

Anti-ProSAP1 and anti-ProSAP2 double-labeling experiments showed that these Abp1-rich synapses do not represent different pools of ProSAP-positive synapses but that individual synapses are positive for both ProSAP isoforms throughout the development of the primary neuronal cultures (Fig. $5 g-l$ ). abundant in the dendritic and postsynaptic compartment.

High-resolution colocalization studies with active zone protein Piccolo as a presynaptic marker (Fig. $6 g-l$ ) show that Abp1 colocalization with Piccolo is partial, as also described by Fenster et al. (2003). Figure $6 g-i$ shows an Abp1-positive dendrite contacted by many Piccolo-positive presynapses. Although partially overlapping with Piccolo, in many cases, the Abp1 immunostaining seems slightly laterally shifted or more extended than the Piccolo staining (see especially Fig. $6 k$ ). Together with the dendritic localization of Abp1 and the presence of Abp1 in postsynaptic spines, this shows that $\mathrm{Abp} 1$ is not restricted to presynapses but is also highly abundant in postsynapses.

ProSAPs are specifically localized to postsynaptic spines and have been shown to be integral components of the PSD (Boeckers 

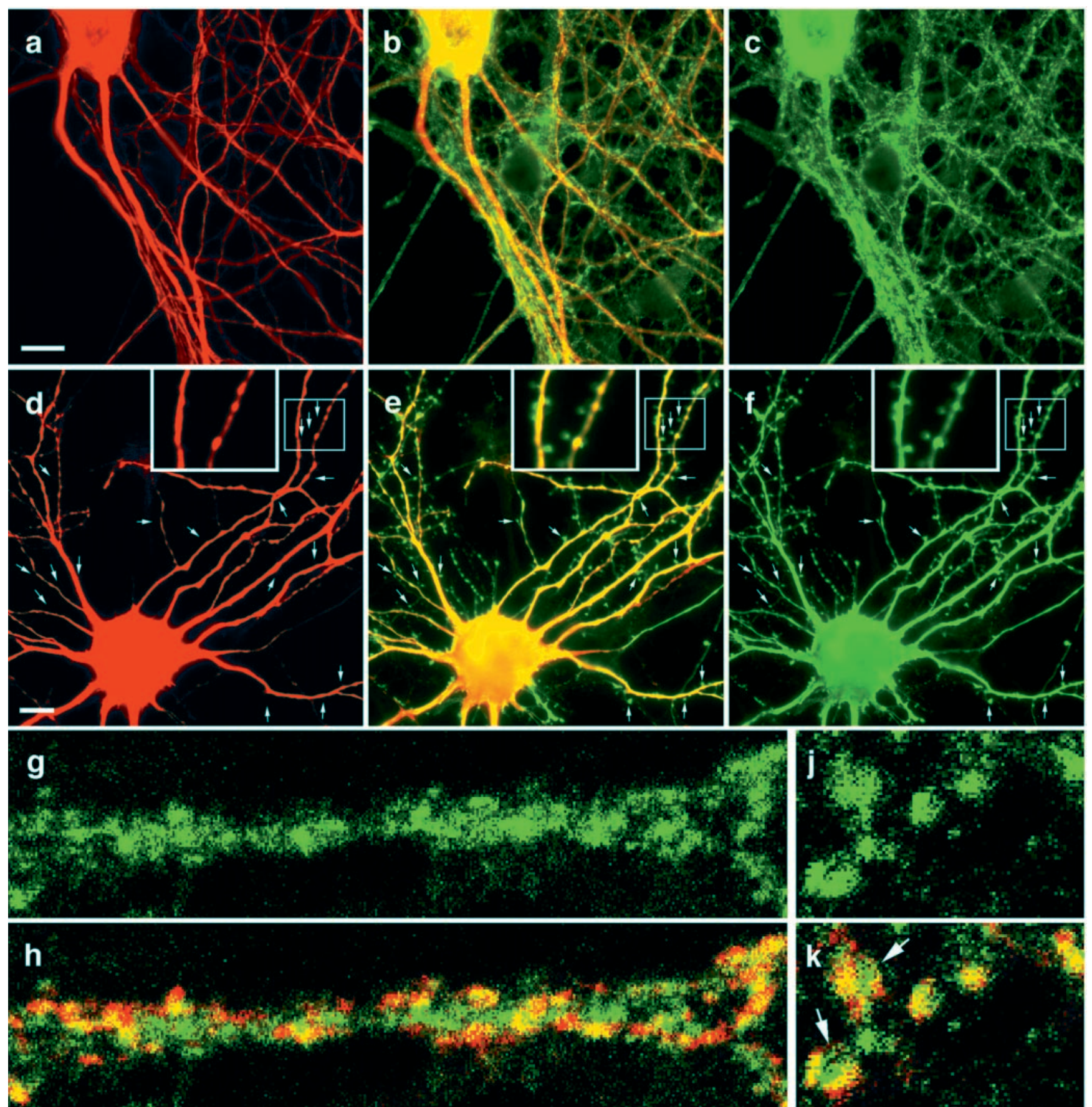


bitis in

its

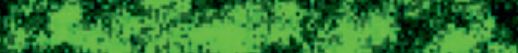

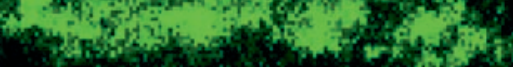
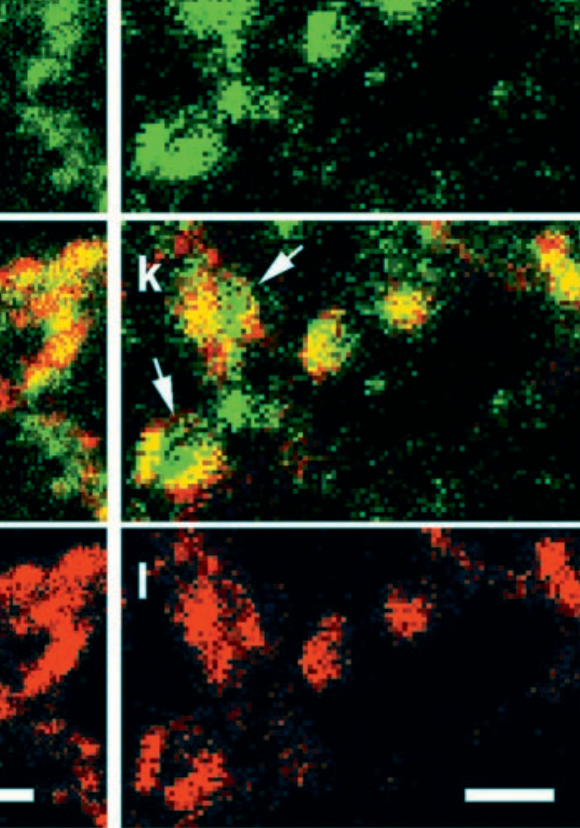

Figure 6. Abp 1 is localized to the dendritic/postsynaptic compartment of hippocampal neurons. $a-c$, Endogenous Abp 1 ( $c$ ) and the dendritic marker protein MAP2 ( $a$ ) were immunolabeled with anti-Abp1 and anti-MAP2 antibodies, respectively. MAP2-positive neurites are also positive for Abp1, as seen in the merged image $(b)$. $d-f$, Primary hippocampal neurons transfected with myc-Abp1 full-length constructs show that myc-Abp1 ( $f$ ) is also localized to MAP2-positive dendrites ( $d$ ), as seen in the merged image (e). Arrows mark examples of myc-Abp1-positive postsynaptic spines. Insets (in $d-f$ ) show an area with dendritic spines (boxed) in a twofold enlargement. Scale bars: $a, d, 10 \mu \mathrm{m} . g-l$, Primary hippocampal neurons were coimmunolabeled with anti-Abp 1 ( $g$, $j)$ and with anti-Piccolo antibodies $(i, l)$. Abp1 and the presynaptic marker Piccolo overlap partially, as well seen in the merged images $(h, k)$. However, the Abp 1 immunostaining often appears slightly laterally shifted and/or more extended than that of Piccolo, a protein that is restricted to active zones of the presynapse ( 2 examples marked by arrows in $k$ ). Scale bars: $i, I, 2 \mu \mathrm{m}$. 


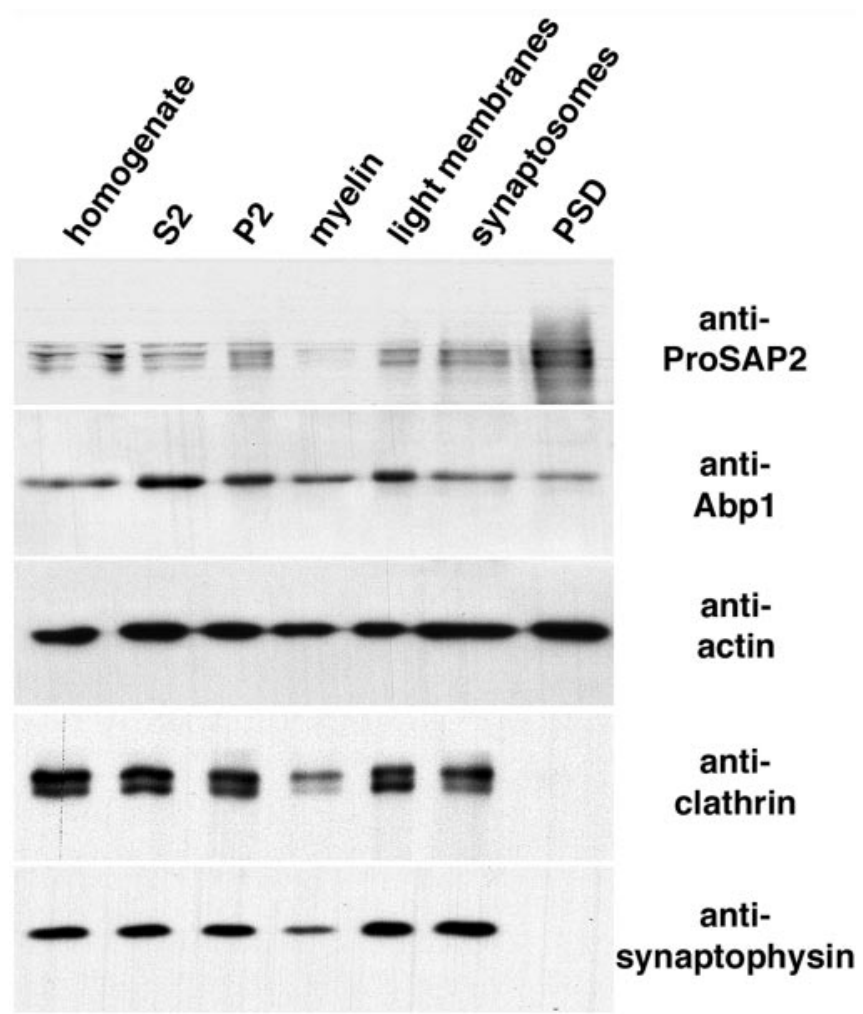

Figure 7. Abp1 is present in the postsynaptic density fraction. Western blots of rat brain homogenate, soluble fraction (S2), crude membrane fraction (P2), myelin fraction (myelin), light membrane fraction (light membranes), synaptosomal fraction (synaptosomes), and the postsynaptic density fraction (PSD). Equal amounts of protein in each fraction were loaded onto SDS-PAGE, blotted to nitrocellulose, and probed with antibodies against ProSAP2, Abp1, actin, clathrin heavy chain, and synaptophysin.

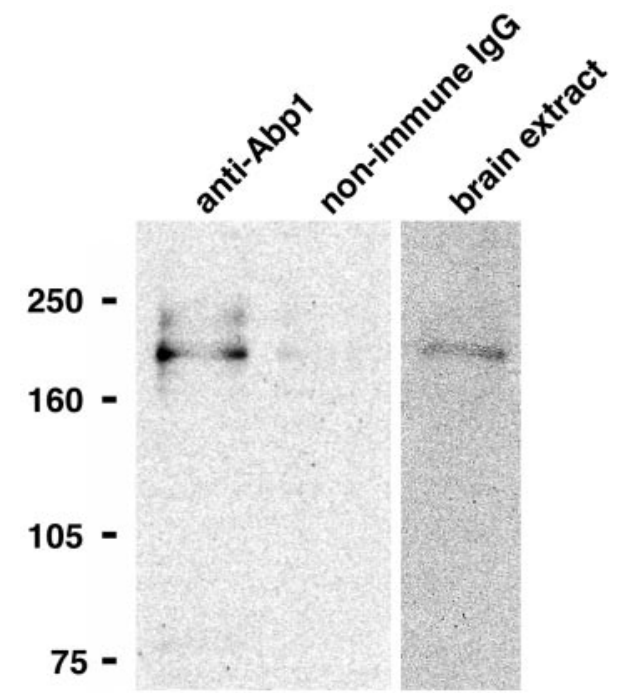

Figure 8. Coimmunoprecipitation of a complex of Abp1 and ProSAP from rat brain. Endogenous ProSAP2, detected by immunoblotting with the anti-ProSAP2 antibodies, was specifically coimmunoprecipitated with Abp1 from rat brain extracts with affinity-purified anti-Abp1 antibodies but not with equal amounts of unrelated guinea pig lgGs. Starting material represents $6 \mu \mathrm{g}$ of brain protein.

et al., 1999a; Naisbitt et al., 1999). We found that Abp1 binds to ProSAPs. We therefore examined whether Abp1 is not only present in ProSAP-positive postsynapses but may also be a component of the synaptic cytoskeleton that is intimately associated with the detergent-insoluble PSD. Immunoblot analyses of PSD preparations revealed that $\mathrm{Abp} 1$ is indeed present in the final PSD fraction that is also strongly enriched for ProSAPs (Fig. 7). However, as for many pancellular proteins, including actin, we did not observe an enrichment of Abp1 in the PSD fraction. This suggests that, in contrast to ProSAPs, Abp1 is not an component of the PSD that is firmly integrated into this subcellular structure but associates with the PSD in a more dynamic manner, as observed for actin as well.

Furthermore, the analyses suggest that Abp1 is not restricted to the PSD but is also localized to other compartments within the cells. This is in agreement with the subcellular distribution of Abp1 shown in Figures 5 and 6 and with the fact that non-neuronal cells also express Abp1. Such non-neuronal cells will contribute especially to the soluble fractions and to the membrane-rich fractions other than synaptic membranes in the subcellular fractionation experiments, but they will for example not contribute to the neuronspecific PSD fraction. Indeed, Abp1 levels were slightly increased in the cytosolic fraction S2 and in the light membrane fraction. However, all other fractions, including synaptosomes and the PSD, contained significant amounts of Abp1 (Fig. 7). In contrast to Abp1, proteins primarily involved in presynaptic membrane trafficking, such as clathrin and the synaptic vesicle protein synaptophysin, were clearly absent from the PSD fraction (Fig. 7).

Endogenous Abp1 and ProSAP can be coimmunoprecipitated from rat brain extracts

To show that endogenous Abp 1 and ProSAPs associate in vivo, we performed coimmunoprecipitation studies. One could argue that anti-ProSAP antibodies are directed against a very stably associated, integral part of the PSD and may simply precipitate the entire synaptic junction, and any coimmunoprecipitated Abpl could then theoretically just be associated with some peripheral actin structures. We therefore decided to use anti-Abp 1 antibodies and probed for ProSAP2. Despite the relatively harsh conditions that were required for successful solubilization of ProSAP2, anti-Abp1 antibodies were able to specifically coimmunoprecipitate ProSAP2 from rat brain extracts in quantities that were readily detectable in the antiAbp1 immunoprecipitates (Fig. 8). This suggests that Abp1 and ProSAP either interact directly or are components of the same, tightly associated protein complex in vivo. The fact that non-immune IgGs used as controls did not precipitate ProSAP2 show that the observed ProSAP2 precipitation is dependent on immunoprecipitated Abpl. Together with the clear evidence for a direct interaction in vitro, the coimmunoprecipitation data indicate that Abpl and ProSAPs also directly interact in vivo.

\section{Abp1 can simultaneously bind ProSAP and perform its actin cytoskeletal functions}

We next wanted to know whether Abp1 would be able to perform its functions in actin dynamics in parallel with its interaction with the PSD scaffold proteins of the ProSAP/Shank family. To address this question, we took advantage of the fact that Abp1 is recruited to the cell periphery and participates in the formation of lamellipodia during activation of certain signaling cascades (Kessels et al., 2000). Cotransfected ProSAP constructs should relocate together with $\mathrm{Abp} 1$ to the leading edge if the interaction is of significant strength in vivo and if the Abp1 SH3 domain that we showed to interact with ProSAPs is not required for the cytoskeletal functions of Abp1. To exclude that the cytosolic GFP-ProSAP fusion protein is giving rise to peripheral localizations attribut- 

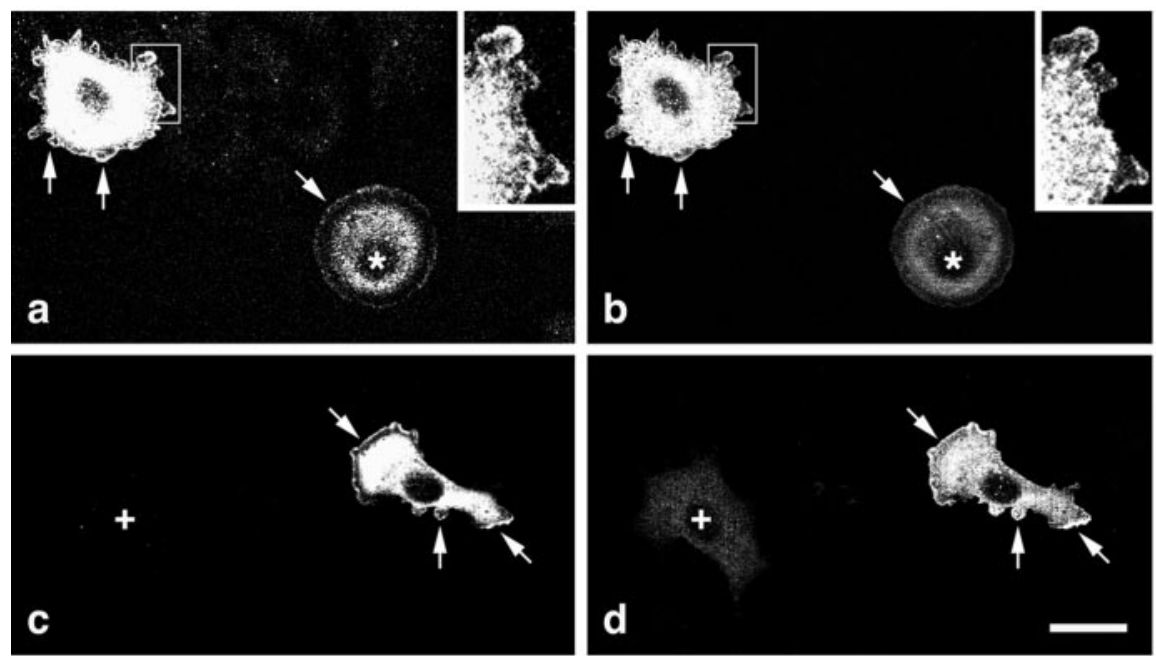

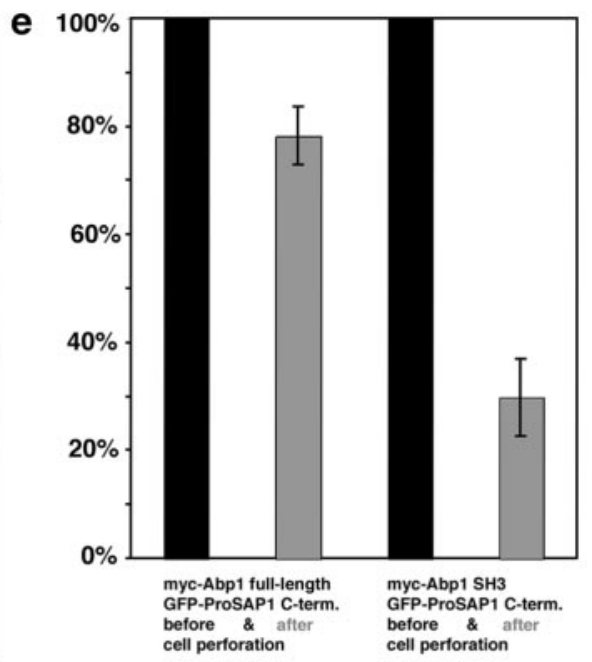

Figure 9. Abp1 can recruit ProSAP fragments to the leading edge of spreading, growth factor-treated NIH3T3 fibroblasts via its association to F-actin. $a-d$, Confocal microscopy of fibroblast cells double transfected with myc-Abp1 full length $(a, c)$ and GFP-ProSAP1C $(b, d)$. The cells, which were activated with growth factors, extend actin-rich lamellipodia that contained F-actin-bound Abp1 at the leading edge (arrows mark some examples in $a$ and $c$ ) and also exhibit ProSAP1C accumulations at these Abp1-rich sites $(b, d)$ that were resistant to perforation. Insets represent high magnifications (2.5-fold) of the boxed areas in $a$ and $b$. Note that cells with low levels of Abp 1 ( ${ }^{*}$ in $a$ and $b$ ) also retained low levels of the ProSAP construct, whereas in cells negative for myc-Abp 1 expression ( + in $($ and $d$ ), almost no GFP-ProSAP1C was retained (d). Scale bar, $20 \mu \mathrm{m}$. $e$, The in vivo association of ProSAP1C is dependent on the SH3 domain of Abp1. Quantification of GFP signal-positive cells double transfected with either myc-Abp1 and GFP-ProSAP1C or with myc-Abp1 SH3 domain and GFP-ProSAP1C after cell perforation. The numbers of GFP-positive cells on coverslips after perforation and fixation (dark gray columns) were compared with those of GFP-positive cells before perforation (defined as 100\%; black columns). Error bars represent SDs of independent assays. Myc-Abp1/GFP-ProSAP1C-double-transfected cells, $78.3 \pm 5.3 \%$ after perforation, 1813 cells scored; myc-Abp1 SH3 domain/GFP-ProSAP1C double-transfected cells, $29.8 \pm$ $7.1 \%$ after perforation, 1074 cells scored.

able to volume effects that may occur when the cells ruffle, we briefly perforated the cells before fixation and used confocal microscopy for the analysis.

Figure 9 shows that, during stimulation with growth factors, Abp1 relocated to the leading edge of the cells (Fig. $9 a, c$ ) and that the GFP-ProSAP1 fusion protein comprising the Abp1 binding site, i.e., GFP-ProSAP1C, can also be found at these actin-rich areas of the cells (Fig. 9b, $d$, arrows). The more Abp1 was expressed (Fig. 9a,c), the more GFP-ProSAP1C fusion protein seemed to be retained (Fig. $9 b, d$ ). In cells not expressing mycAbp1 (Fig. 9c, cross), little GFP signal was retained, but the majority was lost during perforation (Fig. $9 d$, cross). The same was observed for GFP alone used as a control. Only some traces of GFP remained. This was especially true of GFP localized to the nucleus (data not shown). We therefore concluded that GFPProSAP1C was indeed anchored to perforation-resistant cellular structures via Abp1. Because we did not observe any obvious alterations of the morphology of lamellipodia or of other parts of the actin cytoskeleton in transfected cells, it seems that ProSAP binding to the Abp1 SH3 domain does not interfere with the role of Abpl in lamellipodial actin dynamics. The data demonstrate that Abp1 is capable of binding to proteins via its $\mathrm{SH} 3$ domain and performing its cytoskeletal functions simultaneously.

To formally prove that the observed perforation-resistant localization of GFP-ProSAP1C to the actin-rich leading edge is attributable to a retention via the $\mathrm{SH} 3$ domain of $\mathrm{Abp} 1$, we asked whether this retention can be suppressed by an excess of the Abp 1 $\mathrm{SH} 3$ domain. In contrast to the full-length protein, the Abp1 SH3 domain does not have the capability to attach itself to the actin cytoskeleton (Kessels et al., 2000). Therefore, one may predict that, if ProSAP1C is specifically retained by an interaction with the Abp1 SH3 domain in cells expressing full-length Abp1, the ProSAP signal should be lost during perforation of cells expressing both ProSAP1C and the Abp1 SH3 domain, provided that the levels of SH3 domain expression are high enough. We therefore quantified GFP-ProSAP1C-positive cells cotransfected with fulllength myc-Abp1 and those cotransfected with myc-Abp1 SH3 domain before (set to 100\%) and after perforation. Whereas $\sim 80 \%$ of the GFP-ProSAP1C-positive cells in myc-Abp1 fulllength double-transfection experiments remained positive for the GFP signal after perforation, only $30 \%$ of the cells showed some post-perforation GFP signal in myc-Abp1 SH3 domain double-transfection experiments (Fig. 9e). It has to be noted that this value even severely underestimates the obtained effects because only $70.2 \%$ of the GFP-ProSAP1C-transfected cells were also cotransfected with the Abp1 $\mathrm{SH} 3$ domain, as determined in accompanying double-labeling experiments, i.e., in $29.8 \%$ of the cells, no $\mathrm{SH} 3$ domain was present. This number is in extremely good agreement with the number of GFP-ProSAP1C-positive cells found $(29.8 \pm 7.1 \%)$. Thus, the Abp1 SH3 domain strongly suppresses the retention of GFP-ProSAP1C in our assay. This provides direct evidence for the cellular anchoring of the ProSAP fusion protein encompassing the C-terminal region of ProSAP1 being critically dependent on an interaction with the Abp1 SH3 domain and on the ability of Abp1 to interact with the cortical actin cytoskeleton.

\section{The appearance of ProSAPs precedes that of Abp1 at} postsynapses during synaptogenesis

Our data demonstrate that Abp1 has the capability to link the postsynaptic density proteins of the ProSAP family to the actin cytoskeleton. The in situ hybridizations showed coexpression of ProSAPs and Abp1 in developing brains and suggested a role for Abp1 and ProSAP already at early stages of brain development and synaptogenesis. This raised the question, at which time points during the formation of neuronal networks a colocalization of Abp1 and ProSAPs becomes observable and whether ProSAP could be a synaptic anchoring site for Abp1 or whether the actin-binding protein Abp1 may precede ProSAPs at cellular sites destined to become functional synapses, for example, by its 

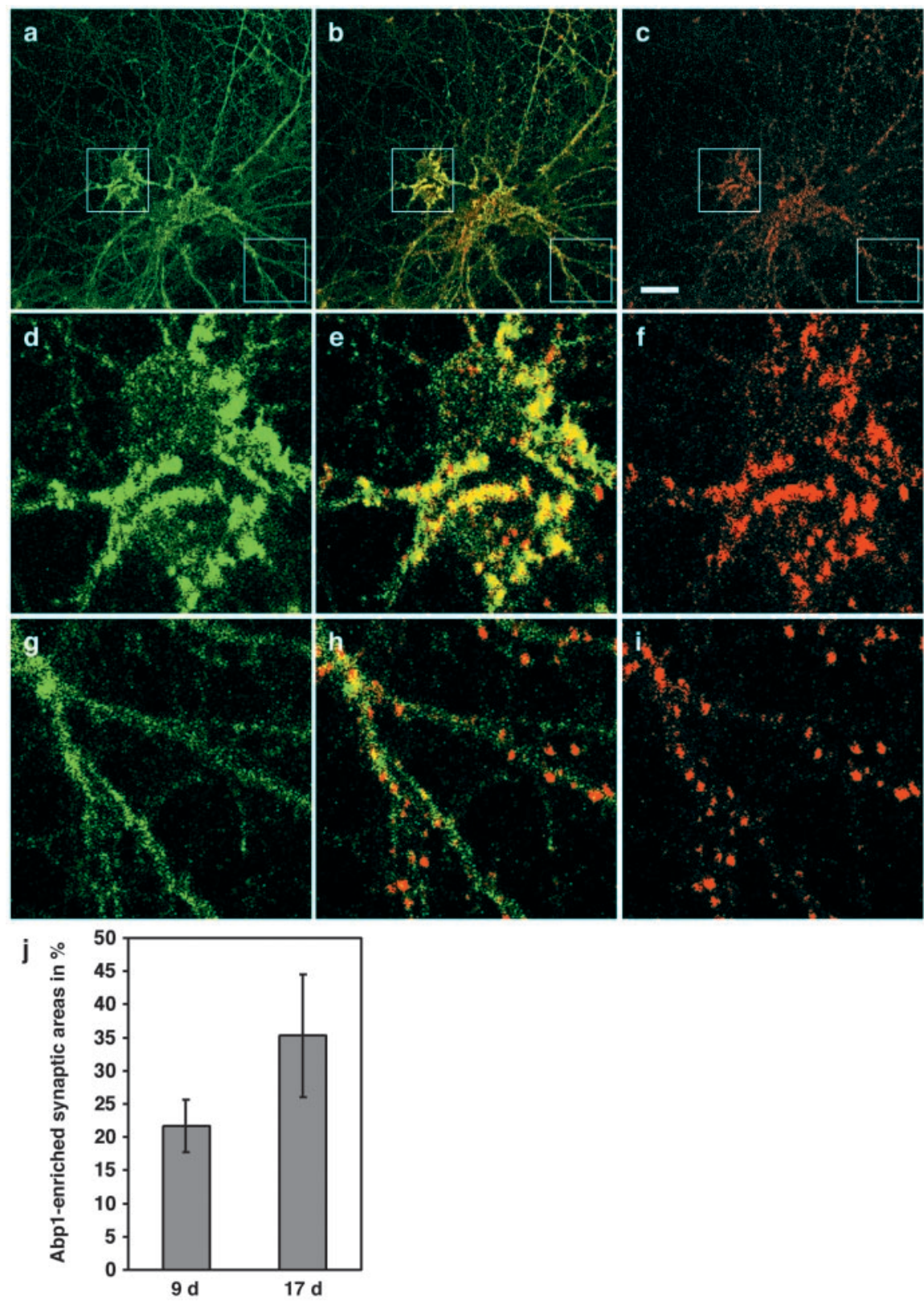

Figure 10. Spatial distribution of ProSAP and Abp1 in developing hippocampal neurons. Cultured rat hippocampal neurons kept in culture for $9 \mathrm{~d}$ were fixed and double stained with anti-Abp1 ( $a$ ) and anti-ProSAP1 antibodies ( $c$ ). The cells displayed Abp1 accumulations in the extended peripheries of cell bodies, as well as a cytosolic and neuritic immunostaining $(a)$. ProSAP1 is coenriched at sites of high Abp1 immunoreactivity in the proximity of the cell bodies ( $c$ ), as well seen in the three cells shown in the merged image $(b)$ and in the 3.5 -fold enlargements of the boxed central areas of the images $(d-f)$. In the extended neurites, ProSAP1 immunoreactivity already displays a concentrated punctate synaptic pattern $(c, i)$, whereas Abp $1(a, g)$ is mainly diffusely distributed throughout the neurites at this stage of development, as best seen in the 3.5-fold enlargements $(g-i)$ of the boxed bottom right areas in $a-c$. Scale bar (in c): $a-c, 20 \mu \mathrm{m}$. j, Quantification of Abp1-enriched synaptic areas, as defined by accumulation of anti-ProSAP1 immunostaining in the periphery of the cell (a $40 \mu \mathrm{m}$ diameter around cell bodies was excluded for these analyses) in immature cultures ( $9 \mathrm{~d}$ ) and in mature cultures (17 d). SDs represent independent images examined. Immature cultures, $21.69 \pm 3.96 \%$ ( 2337 synapses evaluated); mature cultures, $35.27 \pm 9.23 \%$ ( 5635 synapses evaluated). The difference is statistically significant ( $t$ test; $p=0.01779$ ).

capability to accumulate at actin-rich sites, including growth cones.

In a series of coimmunolocalization studies using cells at different time points of neuronal development, we observed that, at early stages of developing neurons in culture, ProSAPs mainly reside in the cell bodies and additionally show a diffuse distribution in neurites (data not shown). Abp1 was also observed mainly in the cell soma and in neurites, and it accumulated at F-actin-rich growth cones at early stages of neuronal development (data not shown).

At day 9 in culture (Fig. 10), i.e., during a period of massive synapse formation, ProSAPs already show a dotted distribution along the neurites. Also at this time, first accumulations of Abp1 at ProSAPrich sites were observed (Fig. 10a-c). Interestingly, these accumulations were observed predominantly in neuritic shafts close to the cell bodies, as displayed in the three cells shown in Figure $10 a-c$. At these sites, the two proteins clearly colocalized (Fig. $10 d-f$ ). The Abp1 signal usually appeared slightly more diffuse than the spatially very restricted ProSAP staining. This may reflect the association and colocalization of Abp1 with F-actin (Kessels et al., 2001), which is also accumulated at these sites (data not shown; cf. Kessels et al., 2001) but not exclusively restricted to PSDs marked by ProSAPs.

In areas more distant from the cell bodies, the periphery, ProSAP-rich puncta were not yet enriched for Abp1 at day 9 in culture (Fig. 10g-i). In some cases, traces of Abp1 staining were observable at these sites (Fig. 10g-i). These traces may, however, merely represent the cytosolic subpool of Abp1. In more mature neurons, in contrast, many of the ProSAP1- and ProSAP2-positive synapses were also found to be stained strongly by anti-Abp1 antibodies (Fig. 5). These analyses showed that synaptic ProSAP accumulation precedes that of Abp1 during the course of neuronal and synaptic development. To quantify this finding, we marked all ProSAP-positive puncta and then merged the images with the anti-Abp1 signal and evaluated the marked areas for the presence of Abp1 accumulations, as described in Materials and Methods. Cell body areas with a diameter of $40 \mu \mathrm{m}$ were excluded from the analyses. ProSAP-marked synaptic areas in the periphery of immature cells are less frequently positive for Abp1 than those of mature neurons (Fig. 10j). This difference is significant $(p=0.01779)$. It should be noted that the quantification may significantly underestimate the postsynaptic Abp1 recruitment during neuronal development because it does not take into account that Abp1-positive synapses in mature neurons usually seem to display higher levels of Abp1 than Abp1-positive synapses in immature neurons.

Because the appearance of ProSAPs precedes that of Abp1 at synaptic sites, they may serve as anchor points for synaptic Abp1 recruitment to PSDs. This does, however, not exclude that also Abp1 (once postsynaptically enriched by ProSAP binding and during its involvement in mediation of local actin dynamics) is 

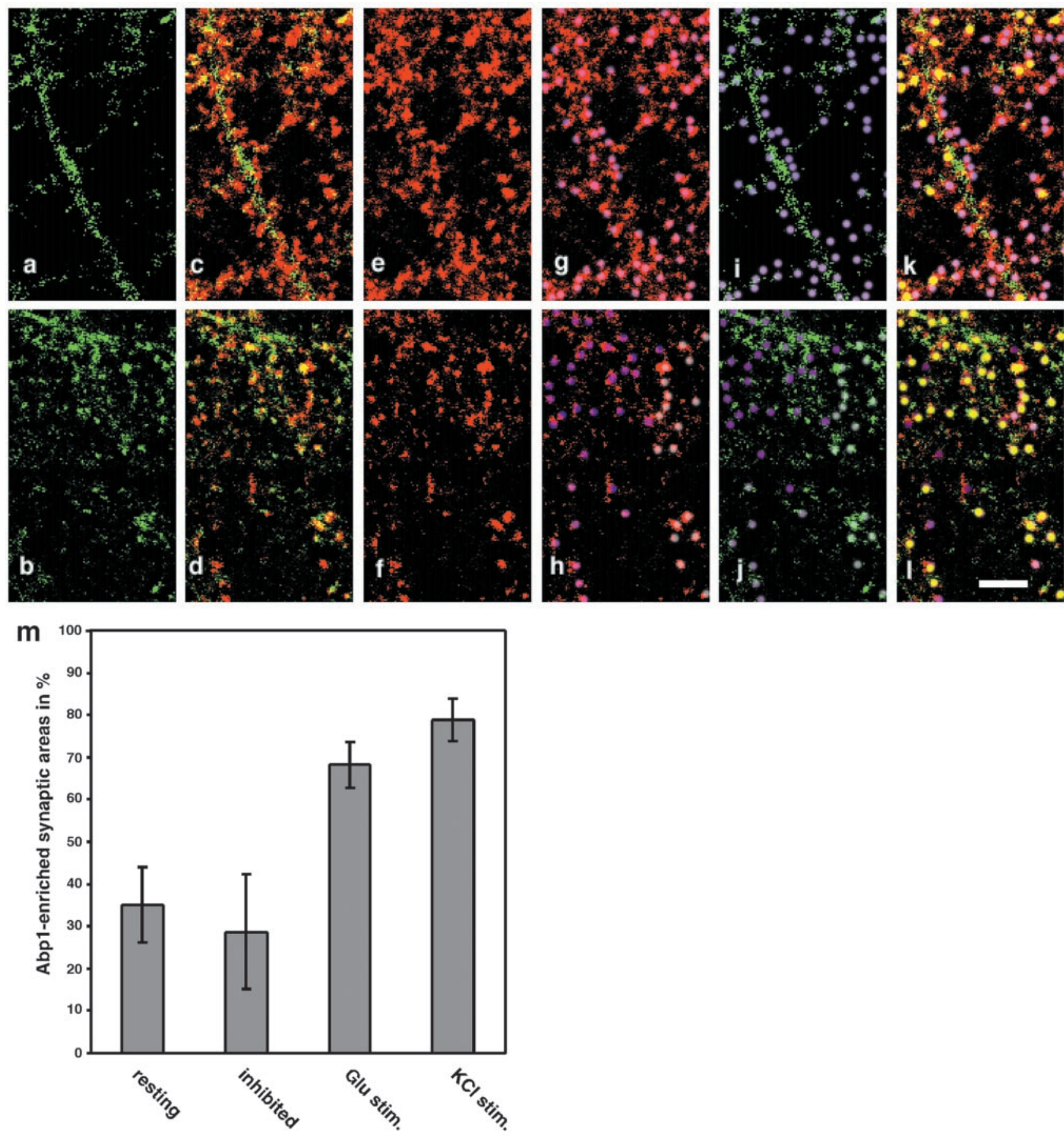

Figure 11. Abp1 accumulates at ProSAP1-positive sites during synaptic stimulation. Untreated $(a, c, e, g, i, k)$, glutamate receptor antagonist-treated, $K C l$-stimulated and glutamate-stimulated $(b, d, f, h, j, l)$ rat hippocampal neurons at day 17 in culture were double stained with anti-Abp 1 (green) and anti-ProSAP1 antibodies (red) and used for quantitative examinations of the percentage of Abp1-enriched postsynaptic areas defined by ProSAP labeling $(m)$. SDs represent five to seven independent, randomly taken images examined. $a-l$, High magnifications of areas of one image for each condition displaying the examination procedure. $a, b$, Immunostaining for Abp1. $c, d$, Merged images of Abp1 and ProSAP1 immunostaining (colocalization appears in different shades of yellow). $e, f$, ProSAP1 immunostainings. ProSAP1 labeling was used to define postsynaptic sites marked by dots in different shades of purple $(g, h)$. These postsynaptic sites defined by ProSAP1 staining were then examined for Abp 1 immunosignals $(i, j)$. Abp 1-positive sites were marked in yellow $(k, I)$ and counted. Scale bar, $5 \mu m$. $m$, Quantification of Abp 1 enrichment at synaptic sites, resting, $35.13 \pm 8.95 \%$ (5938 synapses evaluated in 6 images); glutamate receptor antagonists- treated, $28.72 \pm 13.48 \%$ ( 2544 synapses evaluated in 7 images); glutamate stimulated, $68.23 \pm$ $5.43 \%$ (3162 synapses evaluated in 6 images); and KCl stimulated, $78.83 \pm 5.09 \%$ (1831 synapses evaluated in 5 images). The additional decrease in inhibited cultures compared with resting cultures ( $28.72 \pm 13.48$ vs $35.13 \pm 8.95 \%$ ) is not statistically significant ( $p=0.329$ ), but the differences to both stimulation paradigms are highly significant ( $p=0.00028$, resting vs glutamate stimulated; $p=0.000017$, inhibited vs $\mathrm{KCl}$ stimulated).

capable of recruiting further ProSAPs to spines being remodeled. This is supported by the finding that, although the C-terminal SAM domain of ProSAP1 and ProSAP2 was crucial for synaptic targeting, it was not sufficient. Synaptic targeting of ProSAPs additionally requires a stretch $\mathrm{N}$-terminal of the SAM domain, which corresponds well to the Abp1-binding ProSAP1 construct used in this study (T. M. Boeckers, unpublished observation). Elements critical for postsynaptic ProSAP targeting may thus in- 
clude binding to Abp1 (this study), to cortactin (Du et al., 1998), and/or to dynamin 2 (Okamoto et al., 2001), proteins that all bind to this area within ProSAP molecules. It will be interesting to dissect the synaptic targeting of ProSAP1 and ProSAP2 further. In contrast, Shank1, in which the conserved PXXP-motif of ProSAP1/Shank2 and ProSAP2/Shank3 cannot be found, was reported to rely on the GKAP/SAPAP (guanylate kinase-associated protein/SAP90/PSD-95-associated protein)-binding PDZ (PSD95/DLG/Z01) domain for synaptic targeting (Sala et al., 2001).

\section{Abp1 accumulates at ProSAP-rich sites of primary hippocampal neurons during prolonged synaptic stimulation} The time point at which synapses start to become functional coincided with the first accumulations of Abp1 at ProSAPpositive synapses. We therefore examined whether synaptic signaling may be a trigger for a recruitment of Abp1 to synapses. To do so, the percentage of Abp1-positive postsynaptic areas defined by ProSAP1 immunostaining was examined in untreated mature neurons, in cultures stimulated with glutamate and in cultures stimulated with $\mathrm{KCl}$. Because our first subjective impression was that the extent of Abp1 immunostaining overlapping with that of ProSAP1 was increased during glutamate as well as during $\mathrm{KCl}$ stimulation, we assessed the effects elicited by extended synaptic stimulation quantitatively (Fig. 11). Our examinations revealed that many synaptic areas, as defined by ProSAP1 immunostainings (Fig. 11e-h), were Abp1 enriched in stimulated cultures (Fig. $11 j, l)$, whereas in untreated cultures, there was significant overlap in some areas but very little in other regions (Fig. 11i,k). When the numbers of Abp1-enriched ProSAP1-defined synaptic areas of all images examined were expressed as percentages of the total synaptic areas studied, a significant difference between stimulated and nonstimulated conditions was observed. Whereas in nonstimulated, resting cells, $35.1 \pm 8.95 \%$ of ProSAP1-defined excitatory synapses contained Abp1, $68.23 \pm 5.43$ and $78.83 \pm$ $5.09 \%$ of the synapses are also Abp1 positive in glutamatestimulated and KCl-stimulated cultures, respectively (Fig. $11 \mathrm{~m}$ ). This marked increase of postsynaptic Abp1 levels during stimulation of synaptic transmission prompted us to additionally evaluate cultures, in which synaptic transmission is strongly inhibited. We inhibited NMDA-, AMPA-, and kainite-type glutamate receptors by an incubation with the following antagonists: $50 \mu \mathrm{M}$ (+)-MK-801, $50 \mu \mathrm{M}$ AP-5, $50 \mu \mathrm{M}$ NBQX, and $100 \mu \mathrm{M}$ CNQX. Cultures incubated with this mixture of inhibitors showed an even lower percentage of Abp1-enriched ProSAP-defined synaptic areas than resting cultures. Only $28.72 \pm 13.48 \%$ of the postsynaptic sites were Abp1 immunopositive. Because of the SDs of both quantifications, however, this additional decrease relative to the resting situation is not significant $(p=0.329)$. The differences between the resting or the inhibited conditions on one side and the two different synaptic stimulation paradigms on the other side are all highly significant ( $p$ values range from 0.00028 to 0.000016 ) (Fig. $11 \mathrm{~m}$ ). Our data thus show that Abp1 accumulates at ProSAP1-positive postsynapses during prolonged synaptic transmission mediated by glutamate receptors.

\section{Discussion}

The complex protein network of the PSD is thought to play an important role in formation, maintenance, and functional interconnection of the different molecular cellular machineries within the postsynapse. Although the main PSD scaffolding proteins, including SAP90/PSD-95, SAP97, and the ProSAPs/Shanks, may be known by now (Sheng and Sala, 2001; Hering and Sheng, 2002), the current challenge is to unravel the molecular interconnections of these proteins with the actin cytoskeleton and the machineries for synaptic signal transduction and receptor endocytosis. With Abp1, we identified and characterized a novel F-actin-binding protein that is signal responsive and involved in membrane trafficking (Kessels et al., 2000, 2001; Fucini et al., 2002). Here, we show that Abp1 can associate with both ProSAP1/Shank2 and ProSAP2/Shank3.

The interaction is mediated by the $\mathrm{SH} 3$ domain of Abp1, as evidenced by blot overlays and affinity purifications using this domain. Surprisingly, the highly related endophilin SH3 domain displays no ProSAP binding, suggesting that ProSAP association is characteristic for Abp1. Analysis of ProSAP1/Shank2 and ProSAP2/Shank3 sequences revealed a highly conserved PXXPmotif within the otherwise not well conserved C-terminal parts of the two proteins. In Shank1/synamon/Spank2, this SH3 recognition sequence is not conserved.

The SH3 domain recognition motif (KPPVPPKP) is comprised by an unusual lysine-flanked PXXP sequence that is neither a classical class I nor a class II motif. This motif is supplemented with a partially overlapping second PXXP motif (PPKPKLK). Phage display screens with peptide libraries suggested that the SH3 domain of Abp1 (SH3P7) similar to that of cortactin prefers + PP $\psi$ PpKP-motifs (Yamabhai and Kay, 1997). These preferences match exactly with the conserved Abp1 SH3 domain recognition sequence identified in both ProSAPs. Similarly, the cortactin SH3 domain has been shown to associate with the last 312 amino acids of ProSAP1/CortBP1 (Du et al., 1998). Our blot overlay experiments prove a direct interaction between the Abp1 SH3 domain and the C-terminal part of ProSAPs. This is important because both Abp 1 and ProSAPs have been shown to bind to members of the dynamin protein family (Kessels et al., 2001; Okamoto et al., 2001), and, thus, dynamin could theoretically bridge Abp 1 and ProSAPs in protein complexes.

The in vivo relevance of the Abp1 interaction with ProSAPs is strongly supported by five different experimental lines. First, we demonstrate that, as ProSAPs, Abp1 is a component of the postsynaptic density by biochemical purification of these specialized neuronal structures. Second, we show that Abp1 and ProSAP2 are coexpressed in wide areas of the brain. Both proteins are abundant in the cortex, hippocampus, the caudate putamen, and the cerebellum. ProSAP1 shows a very similar distribution (Boeckers et al., 1999a). Third, our immunofluorescence studies of mature hippocampal neurons demonstrated a colocalization of Abp1 with ProSAP1 and ProSAP2 at postsynapses. The fraction of Abpl-enriched ProSAP-defined postsynapses can reach up to $70-80 \%$ during synaptic stimulation. The observed overlap with both ProSAP isoforms is consistent with our observation that ProSAP1 and ProSAP2 showed virtually identical subcellular localizations. A prominent localization of Abp1 to postsynapses is also consistent with its colocalization with F-actin in these cells (Kessels et al., 2001), which is also known to be enriched postsynaptically (Matus, 2000). Fourth, we demonstrate the presence of Abp1 in the dendritic/postsynaptic compartment by double immunostaining with MAP2. Fifth, we were able to demonstrate that endogenous ProSAP is coimmunoprecipitated from rat brain extracts by Abp1 antibodies. In conclusion, Abp1 and ProSAPs are present in the postsynaptic compartment, are part of the PSD, and can interact in neurons.

Moreover, we find that Abp1 is able to recruit a GFP-ProSAP1 construct to the actin-rich leading edge when cells are activated with growth factor and that Abp1 anchors it to the cytoskeleton, as indicated by the retention in the presence of detergents. This implies that Abp1 can simultaneously perform its actin cytoskel- 
etal functions and associate with ProSAPs in vivo. These data are in line with previous in vitro reconstitution analyses assigning the F-actin binding capabilities of Abp 1 to the N-terminal domains, the ADF-H (actin depolymerizing factor homology) domain (Lappalainen et al., 1998), and the helical domain (Kessels et al., 2000). In contrast, ProSAPs are bound by the C-terminal SH3 domain. An excess of the SH3 domain alone suppressed the recruitment of ProSAP to sites of high actin turnover. These data prove that, also in vivo, the Abp1/ProSAP interaction is based on SH3/PXXP-motif interactions and that the association of ProSAPs with the cytoskeleton can be mediated by Abp1.

Neurons respond to extracellular signals by reorganizing their cortical actin cytoskeleton. Cytoskeletal rearrangements at postsynapses are important in both developing neurons and mature synapses. Activity-mediated reorganizations manifesting in changed spine morphologies and/or dynamics may play a role in synaptic plasticity, which is believed to be the basis for learning and memory (for review, see Matus, 2000; Hering and Sheng, 2002). We here report on the association of the cortical actin cytoskeletal component Abp1 with ProSAPs/Shanks, multidomain proteins highly enriched in the PSD, and show that the localization of Abp1 at ProSAP-positive sites is increased during synaptic stimulation. ProSAPs may thus serve as a starting point for cytoskeletal rearrangements in postsynaptic spines by providing binding sites for cytoskeletal interaction partners, such as Abp1 and cortactin, proteins that are capable of mediating actin dynamics (for review, see Olazabal and Machesky, 2001). Several observations suggest that the actin-binding protein Abp1 may actively participate in actin dynamics. The yeast ortholog of Abp1 has been demonstrated to be an activator of the Arp (actinrelated protein) $2 / 3$ complex promoting actin polymerization in vitro (Goode et al., 2001). Mammalian Abp1 shows a coenrichment with the Arp2/3 complex at the leading edge of lamellipodia of fibroblast cells treated with growth factors (Kessels et al., 2000). Altogether, ProSAP/Shanks may act as key regulators of a dynamic PSD-actin interplay as, in addition to Abpl and cortactin (Du et al., 1998), they can interact with fodrin, another major component of the actin cytoskeleton (Böckers et al., 2001). The fact that overexpression of Shank1 in primary hippocampal cultures altered the morphology of actin-rich dendritic spines underscores this notion (Sala et al., 2001).

Within neuronal cells, ProSAPs are specifically localized to PSDs, whereas Abp1 is present in but not restricted to PSDs. The subcellular distribution of Abp 1 thereby rather parallels that of actin. Although the Abp1 immunoreactivity is enriched in the postsynaptic compartment, as also demonstrated by immuno-EM (Yamazaki et al., 2001), subfractions can be detected elsewhere in the cells, including cell bodies, dendrites, and the presynaptic compartments. In the presynapse, Abp1 seems to create a spatially restricted functional connection between actin and membrane trafficking events by its interaction with the active zone protein Piccolo (Fenster et al., 2003). A functional cross-talk between the cytoskeleton and membrane trafficking machineries in nerve terminals is thought to be important for ensuring high efficiency and accuracy of vesicle formation and recycling (for review, see Qualmann and Kessels, 2002; Gundelfinger et al., 2003).

On the basis of its protein interactions, our current model is that Abp1 might act at different places within in the cell and in distinct cell types to interconnect the dynamic cortical cytoskeleton to machineries mediating other cellular functions, such as synapse organization and membrane trafficking. This proposed role for Abp1 as organizing and connecting element seems not to be restricted to presynapses and postsynapses but to represent a general function of Abp1. Although the expression of Abp1 in the brain is among the highest of all tissues tested, Abp1 appears to be present in all cell types (Kessels et al., 2000). Both biochemical and functional studies have suggested that Abp1 acts as a linker protein between membrane trafficking and the cortical cytoskeleton in non-neuronal cells (Kessels et al., 2000, 2001; MiseOmata et al., 2003). Interestingly, the association of Abp1 with sites of endocytosis was observed to be signal responsive (Kessels et al., 2001) and is thus reminiscent of the relocation of Abp1 to the cortical cytoskeleton (Kessels et al., 2000) and its activitydependent recruitment to ProSAP-positive synapses observed here. Within the endocytic machinery, Abp1 interacts with the GTPase dynamin, which controls the fission reaction and liberates nascent pits from donor membranes (Kessels et al., 2001).

Dynamin mediates endocytosis of AMPA receptors (Carroll et al., 1999; Man et al., 2000), a process that is believed to regulate the surface availability of receptors and to play an important role in long-term potentiation and depression. A multitude of protein-protein interactions allows ProSAPs/Shanks to associate directly or indirectly with different types of glutamate receptors (for review, see Sheng and Kim, 2000; Boeckers et al., 2002). Both Abp1 and ProSAPs/Shanks have been reported to interact with members of the dynamin protein family (Kessels et al., 2001; Okamoto et al., 2001). Interestingly, dynamin2 is a constituent of the PSD fraction (Okamoto et al., 2001). To allow membrane trafficking processes to happen at all, the entire postsynaptic structure has to be dynamic. The requirement of structural organization on one hand and morphological and functional plasticity on the other hand might be brought about best by a dynamic cortical cytoskeleton that is mediated by molecules, such as Abp1, which associate in a signal-responsive manner with dynamic F-actin structures, with scaffold proteins such as ProSAPs/ Shanks, and membrane trafficking machineries.

\section{References}

Böckers TM, Mameza MG, Kreutz MR, Bockmann J, Weise C, Buck F, Richter D, Gundelfinger ED, Kreienkamp H-J (2001) Synaptic scaffolding proteins in rat brain. Ankyrin repeats of the multidomain Shank protein family interact with the cytoskeletal protein alpha-fodrin. J Biol Chem 276:40104-40112.

Bockmann J, Kreutz MR, Gundelfinger ED, Böckers TM (2002) ProSAP/ Shank postsynaptic density proteins interact with insulin receptor tyrosine kinase substrate IRSp53. J Neurochem 83:1013-1017.

Boeckers TM, Kreutz MR, Winter C, Zuschratter W, Smalla K-H, SanmartiVila L, Wex H, Langnaese K, Bockmann J, Garner CC, Gundelfinger ED (1999a) Proline-rich synapse-associated protein-1/cortactin binding protein 1 (ProSAP1/CortBP1) is a PDZ-domain protein highly enriched in the postsynaptic density. J Neurosci 19:6506-6518.

Boeckers TM, Winter C, Smalla K-H, Kreutz MR, Bockmann J, Seidenbecher C, Garner CC, Gundelfinger ED (1999b) Proline-rich synapseassociated proteins ProSAP1 and ProSAP2 interact with synaptic proteins of the SAPAP/GKAP family. Biochem Biophys Res Commun 264:247-252.

Boeckers TM, Bockmann J, Kreutz MR, Gundelfinger ED (2002) ProSAP/ Shank proteins - a family of higher order organizing molecules of the postsynaptic density with an emerging role in human neurological disease. J Neurochem 81:903-910.

Carroll RC, Beattie EC, Xia H, Lüscher C, Altschuler Y, Nicoll RA, Malenka RC, von Zastrow M (1999) Dynamin-dependent endocytosis of ionotrophic glutamate receptors. Proc Natl Acad Sci USA 96:14112-14117.

Dick O, Hack I, Altrock WD, Garner CC, Gundelfinger ED, Brandstätter JH (2001) Localization of the presynaptic cytomatrix protein Piccolo at ribbon and conventional synapses in the rat retina: comparison with Bassoon. J Comp Neurol 15:224-234.

Du Y, Weed SA, Xiong W-C, Marshall TD, Parsons JT (1998) Identification 
of a novel cortactin $\mathrm{SH} 3$ domain-binding protein and its localization to growth cones of cultured neurons. Mol Cell Biol 18:5838-5851.

Ensenat D, Yao Z, Wang XS, Kori R, Zhou G, Lee SC, Tan T-H (1999) A novel src homology 3 domain-containing adaptor protein, HIP-55, that interacts with hematopoietic progenitor kinase 1. J Biol Chem 274:33945-33950.

Fenster SD, Kessels MM, Qualmann B, Wook JC, Nash J, Gundelfinger ED, Garner CC (2003) Interactions between Piccolo and the actin/dynaminbinding protein Abp1 link vesicle endocytosis to presynaptic active zones. J Biol Chem 278:20266-20277.

Fucini RV, Chen J-L, Sharma C, Kessels MM, Stamnes M (2002) Golgi vesicle proteins are linked to the assembly of an actin complex defined by mAbp1. Mol Biol Cell 13:621-631.

Goode BL, Rodal AA, Barnes G, Drubin DG (2001) Activation of the Arp2/3 complex by the actin filament binding protein Abplp. J Cell Biol 153:627-634.

Gundelfinger ED, Kessels MM, Qualmann B (2003) Temporal and spatial coordination of exocytosis and endocytosis. Nat Rev Mol Cell Biol 4:127-139.

Hering H, Sheng M (2002) Dendritic spines: structure, dynamics and regulation. Nat Rev Neurosci 2:880-888.

Kessels MM, Qualmann B (2002) Syndapins integrate N-WASP in receptormediated endocytosis. EMBO J 21:6083-6094.

Kessels MM, Engqvist-Goldstein ÅEY, Drubin DG (2000) Association of mouse actin-binding protein 1 ( $\mathrm{mAbp} 1 / \mathrm{SH} 3 \mathrm{P} 7)$, an src kinase target, with dynamic regions of the cortical actin cytoskeleton in response to Rac1 activation. Mol Biol Cell 11:393-412.

Kessels MM, Engqvist-Goldstein ÅEY, Drubin DG, Qualmann B (2001) Mammalian Abp1, a signal-responsive F-actin-binding protein, links the actin cytoskeleton to endocytosis via the GTPase dynamin. J Cell Biol 153:351-366

Kreutz MR, Bockers TM, Sabel BA, Hulser E, Stricker R, Reiser G (1997) Expression and subcellular localization of p42IP4/centaurin-alpha, a brain-specific, high-affinity receptor for inositol 1,3,4,5-tetrakisphosphate and phosphatidylinositol 3,4,5-trisphosphate in rat brain. Eur J Neurosci 9:2110-2124.

Lappalainen P, Kessels MM, Cope MJTV, Drubin DG (1998) The ADFhomology (ADF-H) domain: a highly exploited actin-binding module. Mol Biol Cell 9:1951-1959.

Man H-Y, Lin JW, Ju WH, Ahmadian G, Liu L, Becker LE, Sheng M, Wang YT (2000) Regulation of AMPA receptor-mediated synaptic transmission by clathrin-dependent receptor internalization. Neuron [Erratum (2001) 29: 307] 25:649-662.

Matus A (2000) Actin-based plasticity in dendritic spines. Science 290:754-758.

Mise-Omata S, Montagne B, Deckert M, Wienands J, Acuto O (2003) Mammalian actin binding protein 1 is essential for endocytosis but not lamellipodia formation: functional analysis by RNA interference. Biochem Biophys Res Commun 301:704-710.

Naisbitt S, Kim E, Tu JC, Xiao B, Sala C, Valtschanoff J, Weinberg RJ, Worely PF, Sheng M (1999) Shank, a novel family of postsynaptic density pro- teins that binds to the NMDA receptor/PSD-95/GKAP complex and cortactin. Neuron 23:569-582.

Okamoto PM, Gamby C, Wells D, Fallon J, Vallee RB (2001) Dynamin isoform-specific interaction with the Shank/ProSAP scaffolding proteins of the postsynaptic density and actin cytoskeleton. J Biol Chem 276:48458-48465.

Olazabal IM, Machesky LM (2001) Abplp and cortactin, new "hand-holds" for actin. J Cell Biol 154:679-682.

Qualmann B, Kelly RB (2000) Syndapin isoforms participate in the receptor-mediated endocytosis and actin organization. J Cell Biol 148:1047-1061.

Qualmann B, Kessels MM (2002) Endocytosis and the cytoskeleton. Int Rev Cytol 220:93-144.

Qualmann B, Roos J, DiGregorio PJ, Kelly RB (1999) Syndapin I, a synapticdynamin-binding protein that associates with the neural Wiskott-Aldrich syndrome protein. Mol Biol Cell 10:501-513.

Qualmann B, Kessels MM, Kelly RB (2000) Molecular links between endocytosis and the actin cytoskeleton. J Cell Biol 150:F111-F116.

Sala C, Piëch V, Wilson NR, Passafaro M, Liu G, Sheng M (2001) Regulation of dendritic spine morphology and synaptic function by Shank and Homer. Neuron 31:115-130.

Sheng M, Kim E (2000) The Shank family of scaffold proteins. J Cell Sci 113:1851-1856

Sheng M, Sala C (2001) PDZ domains and the organization of supramolecular complexes. Annu Rev Neurosci 24:1-29.

Sparks AB, Hoffman NG, McConnell SJ, Fowlkes DM, Kay BK (1996) Cloning of ligand targets: systematic isolation of $\mathrm{SH} 3$ domain-containing proteins. Nat Biotechnol 14:741-744.

Tobaben S, Südhof TC, Stahl B (2000) The G-protein-coupled receptor CL1 interacts directly with proteins of the Shank family. J Biol Chem 275:36204-36210.

Tu JC, Xiao B, Naisbitt S, Yuan JP, Petralia RS, Brakeman P, Doan A, Aakalu VK, Lanahan AA, Sheng M, Worley PF (1999) Coupling of mGluR/ Homer and PSD-95 complexes by Shank family of postsynaptic density proteins. Neuron 23:583-592.

Wyneken U, Smalla K-H, Marengo JJ, Soto D, de la Cerda A, Tischmeyer W, Grimm R, Boeckers TM, Wolf G, Orrego F, Gundelfinger ED (2001) Kainate-induced seizures alter protein composition and N-methyl-Daspartate receptor function of rat forebrain postsynaptic densities. Neuroscience 102:65-74.

Yamabhai M, Kay BK (1997) Examining the specificity of Src homology 3 domain-ligand interactions with alkaline phosphatase fusion proteins. Anal Biochem 247:143-151.

Yamazaki H, Takahashi H, Aoki T, Shirao T (2001) Molecular cloning and dendritic localization of rat SH3P7. Eur J Neurosci 14:998-1008.

Yao I, Hata Y, Hirao K, Deguchi M, Die N, Takeuchi M, Takai Y (1999) Synamon, a novel neuronal protein interacting with synapse-associated protein 90/postsynaptic density-95-associated protein. J Biol Chem 274:27463-27466.

Zitzer H, Richter D, Kreienkamp J (1999) Agonist-dependent interaction of the rat somatostatin receptor subtype 2 with cortactin binding protein J Biol Chem 274:18532-18536. 